\title{
Testing triplet fermions at the electron-positron and electron-proton colliders using fat jet signatures
}

\author{
Arindam Das $\odot,{ }^{1, *}$ Sanjoy Mandal ${ }^{2, \dagger}$ and Tanmoy Modak ${ }^{3, \$}$ \\ ${ }^{1}$ Department of Physics, Osaka University, Toyonaka, Osaka 560-0043, Japan \\ ${ }^{2}$ AHEP Group, Institut de Física Corpuscular, CSIC/Universitat de València, Parc Científic de Paterna, \\ C/ Catedrático José Beltrán, 2 E-46980 Paterna (Valencia), Spain \\ ${ }^{3}$ Department of Physics, National Taiwan University, Taipei 10617, Taiwan
}

(Received 7 May 2020; accepted 22 July 2020; published 5 August 2020)

\begin{abstract}
The addition of $S U(2)_{L}$ triplet fermions of zero hypercharge with the Standard Model (SM) helps to explain the origin of the neutrino mass by the so-called seesaw mechanism. Such a scenario is commonly known as the type-III seesaw model. After the electroweak symmetry breaking, the mixings between the light and heavy mass eigenstates of the neutral leptons are developed and play important roles in the study of the charged and neutral multiplets of the triplet fermions at the colliders. In this article, we study such interactions to produce these multiplets of the triplet fermion at the electron-positron and electron-proton colliders at different center-of-mass energies. We focus on the heavy triplets, for example, having mass in the $\mathrm{TeV}$ scale so that their decay products including the SM, the gauge bosons, or the Higgs boson can be sufficiently boosted, leading to a fat jet. Hence, we probe the mixing between light-heavy mass eigenstates of the neutrinos and compare the results with the bounds obtained by the electroweak precision study.
\end{abstract}

DOI: 10.1103/PhysRevD.102.033001

\section{INTRODUCTION}

The observations of the neutrino oscillation phenomena and the flavor mixing [1-16] give a very strong indication of the existence of the tiny neutrino masses. The existence of the neutrino mass is one of the missing pieces in the Standard Model (SM). Therefore, correctly fitting in the experimental results requires the SM to be extended. From the point of view of the low-energy theory, one can introduce a dimension-5 operator within the SM, which involves the SM Higgs and lepton doublets. Such an operator is known as the very famous Weinberg operator [17], which introduces a violation of the lepton number by two units. The breaking of the electroweak symmetry ensures the generation of the tiny neutrino Majorana masses, which is suppressed by the scale of the dimension-5 operator. In the context of the renormalizable theory, the dimension- 5 operator is naturally generated by the inclusion of the SM singlet right-handed Majorana neutrinos and finally integrating them out. This is called the

\footnotetext{
*arindam.das@ @et.phys.sci.osaka-u.ac.jp †smandal@ific.uv.es

tanmoyy@hep1.phys.ntu.edu.tw
}

Published by the American Physical Society under the terms of the Creative Commons Attribution 4.0 International license. Further distribution of this work must maintain attribution to the author(s) and the published article's title, journal citation, and DOI. Funded by SCOAP . seesaw mechanism [18-23], which can successfully explain the origin of the tiny neutrino mass; however, hitherto, there is no experimental evidence of this simple but extraordinary theoretical aspect. Such nonobservation opens up the pathway for a variety of neutrino mass generation mechanisms.

The type-III seesaw scenario is one of the most simple scenarios in which the SM is extended by an $S U(2)_{L}$ righthanded triplet fermion with zero hypercharge [24] which effectively generates a lepton number-violating dimension5 Weinberg operator. The triplet fermion has neutral and charged multiplets. The neutral component gets involved in the generation of the Majorana mass term for the light neutrinos after the electroweak symmetry breaking, which finally generates the mixing between the light-heavy mass eigenstates as it happens in the canonical or type-I seesaw mechanism. These neutral multiplets can be studied at the energy frontier from their production at the different colliders through the mixing. Apart from the neutral multiplets the charged multiplets can also be produced at the collider in the same fashion; however, the charged multiplets can also be produced directly in pair from the SM gauge interactions in which a wide variety of phenomenological aspects can be studied involving the prompt and nonprompt decay of the triplet fermions [25-32].

The rich theoretical and phenomenological aspects of the type-III seesaw scenario has been explored in different ways. An $S U(5)$ realization of this scenario from the $\mathbf{2 4}$ representation containing both a triplet fermion and a 
singlet fermion has been proposed in Ref. [33]. A supersymmetric realization of the $S U(5)$ grand unified theories for the singlet and triplet has been studied in Refs. [34,35]. In this case, the triplet can reside in the intermediate scale to reproduce the neutrino oscillation data. A nonsupersymmetric implementation of the type-III seesaw in the framework of $S U(5)$ grand unified theory with the inclusion of the adjoint fermionic multiplet has been studied in Ref. [36], which mainly predicts a theory of the light $S U(2)$ triplet fremion with mass at the electroweak scale. It has been mentioned in Ref. [36] that, due to the gauge coupling of the multiplets of the triplets, they can be pair-= produced directly through the Drell-Yan process. Being Majorana in nature, the neutral component of the triplets can show up with a distinct lepton number-violating signature at the collider. The grand unified theory-inspired nonsupersymmetric and supersymmetric renormalizable $S U(5)$ frameworks to study the origin of neutrino masses generated by type-III and type-I seesaw mechanisms have been discussed in Refs. [37,38]. Collider phenomenology of the heavy triplet fermions from such models has been studied in Refs. [39-41]. An inverse seesaw realization under the type-III seesaw framework has been studied in Ref. [42] involving a singlet hyperchargeless fermion. Another type of type-III seesaw was studied in Ref. [43], in which an extra U(1) gauge group has been introduced to the SM under the anomaly-free scenario [44-46].

The renormalization group evolution of the effective neutrino mass matrix in the $S U(2)_{L}$ triplet fermion extended SM with emphasis on the threshold effects has been studied in Ref. [47]. In the type-III seesaw scenario with degenerate heavy triplets, the impact of the renormalization group evolution in the context of perturbativity bounds and vacuum stability of the scalar potential has been studied in Ref. [48]. Adding two $S U(2)_{L}$ triplet fermions with zero hypercharge has been studied to probe electroweak vacuum stability with the nonzero neutrino mass, naturalness, and lepton flavor violation in Ref. [49]. A simple realization of the triplet fermion under $S U(2)_{L}$ with a general U(1) extension of the SM has been discussed in Ref. [50], in which a neutral gauge boson $\left(Z^{\prime}\right)$ plays an important role for the triplet fermion in addition to the SM gauge boson mediated processes. Over the years at different center-of-mass energies and integrated luminosities, the LHC has also been searching for the fermions triplet under the $S U(2)_{L}$ group in Refs. [51-59] from SM gauge mediated processes using different flavor structures of the Yukawa interaction involving the triplet fermion, SM lepton, and Higgs doublets. The latest result from the LHC put strong bounds on the triplets lighter than 880 [57] and $590 \mathrm{GeV}$ [59]. This leads us to find a strategy for the heavy triplet fermion search at the energy frontier in the future.

Apart from the collider searches, lepton flavor-violating [60] and nonunitarity effects in the type-III model have been studied in Refs. [61,62]. In this context, it is necessary to mention that such studies have been performed in the context of the type-I seesaw scenario [63-71]. The bounds on the light heavy neutrino mixings from the eletroweak precision data (EWPD) have been studied in Refs. [72,73], which can be considered as the upper limits to constrain the limits on the mass-mixing plane for the triplets.

In this paper, we study the production of the $\mathrm{TeV}$ scale triplet fermions at electron-positron $\left(e^{-} e^{+}\right)$collider. At the $e^{-} e^{+}$collider, the production of the neutral multiplet of the triplet fermion will take place through the $s$ - and $t$-channel processes in association with the SM leptons. Similarly, the charged multiplets of the triplet can be produced from the $s$ - and $t$-channel processes in association with the SM leptons; however, they can be produced in pair from the $s$-channel process. If the triplets are in the $\mathrm{TeV}$ scale and heavy, they can sufficiently boost the decay products such as SM gauge bosons $(W, Z)$ and the SM Higgs $(h)$, which can further produce fat jets from the leading hadronic decay modes. Such a fat jet signature can have a distinctive nature to separate the signal from the SM backgrounds. Following this, we study the allowed parameter space in the massmixing plane from the associated production of the triplets with SM leptons. We also study the significance of several final states coming from the pair production of the charged multiplets. At the $e^{+} e^{-}$collider, we consider two centerof-mass energies $\sqrt{s}=1$ and $3 \mathrm{TeV}$ with integrated luminosities at $\mathcal{L}=1,3$, and $5 \mathrm{ab}^{-1}$. In this context, we mention that in the seesaw scenario a variety of final states has been studied for the linear collider in the literatures [74-84]. We also study the production of the neutral and charged multiplet of the triplet fermion at the electronproton $\left(e^{-} p\right)$ collider at different center-of-mass energies, $\sqrt{s}=1.3,1.8$, and $3.46 \mathrm{TeV}$. As we are concentrating in the heavy mass range of the triplet fermions, we expect that their decay products will be sufficiently boosted to probe the mass-mixing plane. To do this, we fix the collider energy at $\sqrt{s}=3.46 \mathrm{TeV}$ (FCC-he) with the luminosity $\mathcal{L}=1,3$, and $5 \mathrm{ab}^{-1}$, respectively. Studies on the $e^{-} p$ collider considering the seesaw scenario have been performed in Refs. [85-87]. In both of these colliders, to identify the fat jet from the boosted decay products of the heavy triplet fermions, we study the signals and the SM backgrounds. In this context, we mention that in the $e^{-} p$ collider testing the Majorana nature of a fermion could be very interesting because it can show a lepton numberviolation signature distinctively. Such a process for the seesaw scenario has been studied in Refs. [81,84].

This paper is organized as follows. In Sec. II, we discuss the model and the interactions of the triplet fermions with the SM particles. In Sec. III, we study various production processes of the charged and neutral multiplets of the triplet fermion at the $e^{-} e^{+}$and $e^{-} p$ colliders. In Sec. IV, we discuss the complete collider study of various possible final states and calculate the bounds on the mixing angles in the 
mass-mixing plane. We also compare the results with the existing constraints. In Sec. V, we discuss the calculated bounds. Finally, in Sec. VI, we conclude.

\section{MODEL}

The type-III seesaw model is a simple extension of the $\mathrm{SM}$ with an $S U(2)_{L}$ triplet fermion $(\tilde{\Sigma})$ with zero hypercharge. A three-generation triplet fermion of this kind can be introduced in this model to generate the neutrino mass. For simplicity, we suppress the generation indices. The relevant part of the Lagrangian can be written as

$$
\begin{aligned}
\mathcal{L}_{\text {int }}= & \mathcal{L}_{\mathrm{SM}}+\operatorname{Tr}\left(\overline{\tilde{\Sigma}} i \gamma^{\mu} D_{\mu} \tilde{\Sigma}\right)-\frac{1}{2} M_{\Sigma} \operatorname{Tr}\left(\overline{\tilde{\Sigma}} \tilde{\Sigma}^{c}+\overline{\tilde{\Sigma}} \bar{\Sigma} \tilde{\Sigma}\right) \\
& -\sqrt{2}\left(\overline{\ell_{L}} Y^{\dagger} \tilde{\Sigma} H+H^{\dagger} \overline{\tilde{\Sigma}} Y \ell_{L}\right),
\end{aligned}
$$

where the first term is the kinetic interaction of the triplet and $D_{\mu}$ represents the covariant derivative. $\mathcal{L}_{\mathrm{SM}}$ is relevant part of the SM Lagrangian. In the second term of Eq. (1), $M_{\Sigma}$ represents the triplet mass parameter. For simplicity, we consider $M_{\Sigma}$ as a real parameter, and the triplets are degenerate in nature. Therefore, $M_{\Sigma}$ is a real diagonal matrix. $Y$ in the third term of Eq. (1) is the Yukawa coupling between the SM lepton doublet $\left(\ell_{L}\right)$, SM Higgs doublet $(H)$, and the triplet fermion $(\tilde{\Sigma})$. In this analysis, we represent the relevant SM candidates in the following way:

$$
\ell_{L}=\left(\begin{array}{c}
\nu_{L} \\
e_{L}
\end{array}\right) \quad \text { and } \quad H=\left(\begin{array}{c}
\phi^{0} \\
\phi^{-}
\end{array}\right)
$$

After the symmetry breaking, $\phi^{0}$ acquires the vacuum expectation value, and we can express it as $\phi^{0}=\frac{v+h}{\sqrt{2}}$ with $v=246 \mathrm{GeV}$. The explicit form of $\tilde{\Sigma}$ and its charge conjugate $\tilde{\Sigma}^{c} \equiv C \tilde{\tilde{\Sigma}}^{T}$ ( $C$ is the charge conjugation operator) are given by

$$
\begin{aligned}
\tilde{\Sigma} & =\left(\begin{array}{cc}
\Sigma^{0} / \sqrt{2} & \Sigma^{+} \\
\Sigma^{-} & -\Sigma^{0} / \sqrt{2}
\end{array}\right) \text { and } \\
\tilde{\Sigma}^{c} & =\left(\begin{array}{cc}
\Sigma^{0 c} / \sqrt{2} & \Sigma^{-c} \\
\Sigma^{+c} & -\Sigma^{0 c} / \sqrt{2}
\end{array}\right),
\end{aligned}
$$

whereas $D_{\mu}$ is given by

$$
D_{\mu}=\partial_{\mu}-i \sqrt{2} g\left(\begin{array}{cc}
\frac{W_{\mu}^{3}}{\sqrt{2}} & W_{\mu}^{+} \\
W_{\mu}^{-} & -\frac{W_{\mu}^{3}}{\sqrt{2}}
\end{array}\right) .
$$

To study the mixing between the SM charged leptons and the charged multiplets of the triplet fermions, it is convenient to express the 4 degrees of freedom of each of the charged multiplets of the triplet fermions in terms of a single Dirac spinor as $\Sigma=\Sigma_{R}^{-}+\Sigma_{R}^{+c}$. The neutral multiplets of the triplet fermions are two component fermions. Therefore, they have the 2 degrees of freedom. Finally mixing with the SM light neutrinos, these neutral fermions generate tiny neutrino mass through the seesaw mechanism after the electroweak symmetry breaking. With this convention, we rewrite the Eq. (1) as

$$
\begin{aligned}
\mathcal{L}_{\text {int }}= & \bar{\Sigma} i \not \Sigma+\overline{\Sigma_{R}^{0}} i \not \Sigma_{R}^{0}-\bar{\Sigma} M_{\Sigma} \Sigma-\left(\overline{\Sigma_{R}^{0}} \frac{M_{\Sigma}}{2} \Sigma_{R}^{0 c}+\text { H.c. }\right) \\
& -\left(\phi^{0} \overline{\Sigma_{R}^{0}} Y_{\Sigma} \nu_{L}+\sqrt{2} \phi^{0} \bar{\Sigma} Y e_{L}+\phi^{+} \overline{\Sigma_{R}^{0}} Y e_{L}\right. \\
& \left.-\sqrt{2} \phi^{+} \overline{\nu_{L}^{c}} Y^{T} \Sigma+\text { H.c. }\right) \\
& -g W_{\mu}^{3} \bar{\Sigma} \gamma^{\mu} \Sigma .
\end{aligned}
$$

After the electroweak symmetry breaking, we can derive the mass matrix for charged and neutral sectors. The mass term of the charged leptons shows the usual nature of the Dirac particles, and it can be written as

$$
\begin{aligned}
-\mathcal{L}_{\text {mass }}^{\text {charged }}= & \left(\begin{array}{ll}
\bar{e}_{R} & \bar{\Sigma}_{R}
\end{array}\right)\left(\begin{array}{cc}
m_{\ell} & 0 \\
Y v & M_{\Sigma}
\end{array}\right)\left(\begin{array}{c}
e_{L} \\
\Sigma_{L}
\end{array}\right) \\
& +\left(\begin{array}{ll}
\bar{e}_{L} & \bar{\Sigma}_{L}
\end{array}\right)\left(\begin{array}{cc}
m_{\ell} & Y^{\dagger} v \\
0 & M_{\Sigma}
\end{array}\right)\left(\begin{array}{c}
e_{R} \\
\Sigma_{R}
\end{array}\right),
\end{aligned}
$$

where $m_{\ell}$ represents the Dirac mass of the SM charged lepton coming from the well-known SM Lagrangian. Similarly, the mass term for the neutral fermions can be given as

$$
\begin{aligned}
-\mathcal{L}_{\text {mass }}^{\text {neutral }}= & \left(\begin{array}{ll}
\overline{\nu_{L}} & \overline{\Sigma_{R}^{0 c}}
\end{array}\right)\left(\begin{array}{cc}
0 & Y^{\dagger} \frac{v}{2 \sqrt{2}} \\
Y^{*} \frac{v}{2 \sqrt{2}} & \frac{M_{\Sigma}}{2}
\end{array}\right)\left(\begin{array}{c}
\nu_{L}^{c} \\
\Sigma_{R}^{0}
\end{array}\right) \\
& +\left(\begin{array}{ll}
\overline{\nu_{L}^{c}} & \overline{\Sigma_{R}^{0}}
\end{array}\right)\left(\begin{array}{cc}
0 & Y^{T} \frac{v}{2 \sqrt{2}} \\
Y \frac{v}{2 \sqrt{2}} & \frac{M_{\Sigma}}{2}
\end{array}\right)\left(\begin{array}{c}
\nu_{L} \\
\Sigma_{R}^{0 c}
\end{array}\right) .
\end{aligned}
$$

In case of a Dirac mass, the charged lepton mass matrix can be diagonalized by a biunitary transformation, which can be written as

$$
\left(\begin{array}{c}
e_{L} \\
\Sigma_{L}
\end{array}\right)=\mathcal{U}_{L}\left(\begin{array}{c}
e_{L}^{\prime} \\
\Sigma_{L}^{\prime}
\end{array}\right) \quad \text { and } \quad\left(\begin{array}{c}
e_{R} \\
\Sigma_{R}
\end{array}\right)=\mathcal{U}_{R}\left(\begin{array}{c}
e_{R}^{\prime} \\
\Sigma_{R}^{\prime}
\end{array}\right)
$$

The transformation matrices in Eq. (8) can be written as

$$
\begin{aligned}
\mathcal{U}_{L} & =\left(\begin{array}{cc}
1-\epsilon & Y^{\dagger} M_{\Sigma}^{-1} v \\
-M_{\Sigma}^{-1} Y v & 1-\epsilon^{\prime}
\end{array}\right) \text { and } \\
\mathcal{U}_{R} & =\left(\begin{array}{cc}
1 & m_{\ell} Y^{\dagger} M_{\Sigma}^{-2} v \\
-M_{\Sigma}^{-2} Y m_{\ell} v & 1
\end{array}\right) .
\end{aligned}
$$

The symmetric neutral lepton mass matrix can be diagonalized by a single unitary, which can be written as 


$$
\left(\begin{array}{c}
\nu_{L} \\
\Sigma_{R}^{0 c}
\end{array}\right)=\mathcal{V}\left(\begin{array}{c}
\nu_{L}^{\prime} \\
\Sigma_{R}^{\prime 0 c}
\end{array}\right)
$$

The transformation matrix in Eq. (10) can be written as

$$
\mathcal{V}=\left(\begin{array}{cc}
\left(1-\frac{\epsilon}{2}\right) U_{\mathrm{PMNS}} & \frac{Y^{\dagger} M_{\Sigma}^{-1} v}{\sqrt{2}} \\
\frac{-M_{\Sigma}^{-1} Y v}{\sqrt{2}}\left(1-\frac{\epsilon}{2}\right) U_{\mathrm{PMNS}} & 1-\frac{\epsilon^{\prime}}{2}
\end{array}\right)
$$

In the above expressions, $\epsilon=\frac{v^{2}}{2} Y^{\dagger} M_{\Sigma}^{-2} Y, \epsilon^{\prime}=\frac{v^{2}}{2} M_{\Sigma}^{-1} Y Y^{\dagger} M_{\Sigma}^{-1}$, and $U_{\text {PMNS }}$ (Pontecorvo-Maki-Nakagawa-Sakata) is the lowest-order unitary neutrino mixing matrix from Refs. [28,60] in accordance with Ref. [61]. According to Refs. [28,60], $\epsilon$ and $\epsilon^{\prime}$ are the small quantities where we can neglect the effects of the higher powers (above 1 ) of them in the calculations. For a three-generation case, $\mathcal{U}_{L}, \mathcal{U}_{R}$, and $\mathcal{V}$ are the $6 \times 6$ unitary matrices. The neutrino mass can be generated by the seesaw mechanism after the diagonalization of the neutral lepton mass matrix in Eq. (7), can be written as

$$
m_{\nu}=-\frac{v^{2}}{2} Y^{T} M_{\Sigma}^{-1} Y
$$

Because of Eqs. (9) and (11), a mixing parameter between the SM lepton and the triplet is generated, which affects the interaction of the triplet fermion with the SM leptons through the $W, Z$, and $H$ bosons. The modified charged current (CC) interaction can be written as

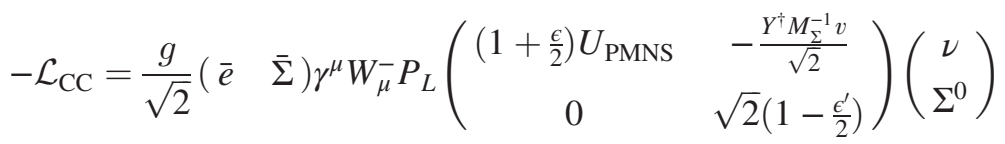

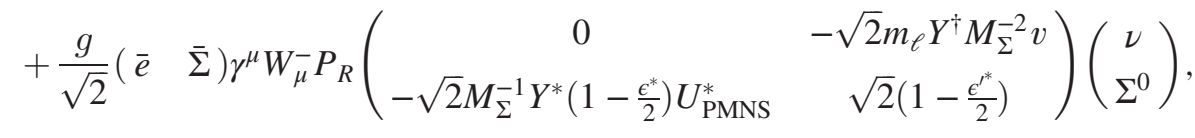

and the modified neutral current (NC) interaction for the charged sector can be written as

$$
\begin{aligned}
-\mathcal{L}_{\mathrm{NC}}^{1}= & \frac{g}{\cos \theta_{W}}\left(\begin{array}{ll}
\bar{e} & \bar{\Sigma}
\end{array}\right) \gamma^{\mu} Z_{\mu} P_{L}\left(\begin{array}{cc}
\frac{1}{2}-\cos ^{2} \theta_{W}-\epsilon & \frac{Y^{\dagger} M_{\Sigma}^{-1} v}{2} \\
\frac{M_{\Sigma}^{-1} Y v}{2} & \epsilon^{\prime}-\cos ^{2} \theta_{W}
\end{array}\right)\left(\begin{array}{l}
e \\
\Sigma
\end{array}\right) \\
& +\frac{g}{\cos \theta_{W}}\left(\begin{array}{ll}
\bar{e} & \bar{\Sigma}
\end{array}\right) \gamma^{\mu} Z_{\mu} P_{R}\left(\begin{array}{cc}
1-\cos ^{2} \theta_{W} & m_{\ell} Y^{\dagger} M_{\Sigma}^{-2} v \\
M_{\Sigma}^{-2} Y m_{\ell} v & -\cos ^{2} \theta_{W}
\end{array}\right)\left(\begin{array}{l}
e \\
\Sigma
\end{array}\right) .
\end{aligned}
$$

The modified NC interaction for the neutral sector of the leptons can be written as

$$
-\mathcal{L}_{\mathrm{NC}}^{2}=\frac{g}{2 \cos \theta_{W}}\left(\begin{array}{ll}
\bar{\nu} & \overline{\Sigma^{0}}
\end{array}\right) \gamma^{\mu} Z_{\mu} P_{L}\left(\begin{array}{cc}
1-U_{\mathrm{PMNS}}^{\dagger} \epsilon U_{\mathrm{PMNS}} & \frac{U_{\mathrm{PMNS}}^{\dagger} Y^{\dagger} M_{\Sigma}^{-1} v}{\sqrt{2}} \\
\frac{M_{\Sigma}^{-1} Y_{\Sigma} U_{\mathrm{PMNS}} v}{\sqrt{2}} & \epsilon^{\prime}
\end{array}\right)\left(\begin{array}{c}
\nu \\
\Sigma^{0}
\end{array}\right),
$$

where $\theta_{W}$ is the Weinberg angle or weak mixing angle. The complete $\mathrm{NC}$ interaction can be written as $\mathcal{L}_{\mathrm{NC}}=\mathcal{L}_{\mathrm{NC}}^{1}+\mathcal{L}_{\mathrm{NC}}^{2}$. Finally, we write the interaction Lagrangian of the SM leptons and charged and neutral multiplets of the triplet fermions with the SM Higgs $(h)$ boson. The interaction between the charged sector and the $h$ can be written as

$$
\begin{aligned}
-\mathcal{L}_{H}^{1}= & \frac{g}{2 M_{W}}\left(\begin{array}{ll}
\bar{e} & \bar{\Sigma}
\end{array}\right) h P_{L}\left(\begin{array}{cc}
-\frac{m_{\ell}}{v}(1-3 \epsilon) & m_{\ell} Y^{\dagger} M_{\Sigma}^{-1} \\
Y(1-\epsilon)+M_{\Sigma}^{-2} Y m_{\ell}^{2} & Y Y^{\dagger} M_{\Sigma}^{-1} v
\end{array}\right)\left(\begin{array}{c}
e \\
\Sigma
\end{array}\right) \\
& +\frac{g}{2 M_{W}}\left(\begin{array}{ll}
\bar{e} & \bar{\Sigma}
\end{array}\right) P_{R}\left(\begin{array}{cc}
-\frac{m_{\ell}}{v}\left(1-3 \epsilon^{*}\right) & M_{\Sigma}^{-1} Y^{\dagger} m_{\ell} \\
\left(1-\epsilon^{*}\right) Y^{\dagger}+m_{\ell}^{2} Y_{\Sigma}^{\dagger} M_{\Sigma}^{-2} & M_{\Sigma}^{-1} Y Y^{\dagger} v
\end{array}\right)\left(\begin{array}{c}
e \\
\Sigma
\end{array}\right)
\end{aligned}
$$

and that between the neutral sector and the SM Higgs can be written as 


$$
\begin{aligned}
-\mathcal{L}_{H}^{2}= & \left(\begin{array}{ll}
\bar{\nu} & \overline{\Sigma^{0}}
\end{array}\right) h P_{L}\left(\begin{array}{cc}
\frac{\sqrt{2} m_{\nu}}{v} & U_{\mathrm{PMNS}}^{T} m_{\nu} Y^{\dagger} M_{\Sigma}^{-1} \\
\left(Y-\frac{Y \epsilon}{2}-\frac{\epsilon^{\prime T} Y}{2}\right) U_{\mathrm{PMNS}} & \frac{Y Y^{\dagger} M_{\Sigma}^{-1} v}{\sqrt{2}}
\end{array}\right)\left(\begin{array}{c}
\nu \\
\Sigma^{0}
\end{array}\right) \\
& +\left(\begin{array}{cc}
\bar{e} & \overline{\Sigma^{0}}
\end{array}\right) P_{R}\left(\begin{array}{cc}
\frac{\sqrt{2} m_{\nu}}{v} & M_{\Sigma}^{-1} Y m_{\nu} U_{\mathrm{PMNS}}^{*} \\
U_{\mathrm{PMNS}}^{*}\left(Y^{\dagger}-\frac{\epsilon^{*} Y^{\dagger}}{2}-\frac{Y^{\dagger} \epsilon^{* *} Y}{2}\right) & \frac{M_{\Sigma}^{-1} Y Y^{\dagger} v}{\sqrt{2}}
\end{array}\right)\left(\begin{array}{c}
\nu \\
\Sigma^{0}
\end{array}\right) .
\end{aligned}
$$

The quantities $m_{\ell}$ and $m_{\nu}$ are the SM charged lepton and tiny light neutrino mass matrices which are real and diagonal, too. The effect of these masses in the collider study will be negligible. Therefore, in our further analyses, we do not consider the effects coming from them. The complete Higgs interaction can be written as $\mathcal{L}_{H}=$ $\mathcal{L}_{H}^{1}+\mathcal{L}_{H}^{2}$. We want to make a comment that the general expressions calculated independently from Eqs. (6)-(17) in this section match exactly with the expressions given in Refs. [28,60]. The charged multiplets of the triplet fermions can interact with photons $\left(A_{\mu}\right)$. The corresponding Lagrangian derived from Eq. (5) can be written as

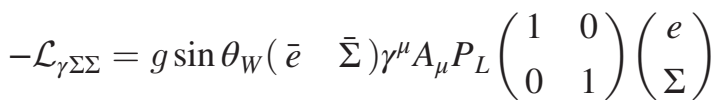

$$
\begin{aligned}
& +g \sin \theta_{W}\left(\begin{array}{ll}
\bar{e} & \bar{\Sigma}
\end{array}\right) \gamma^{\mu} A_{\mu} P_{R}\left(\begin{array}{ll}
1 & 0 \\
0 & 1
\end{array}\right)\left(\begin{array}{l}
e \\
\Sigma
\end{array}\right) .
\end{aligned}
$$

At this point, we want to mention that in the rest of the text we express the light-heavy mixing by $V_{\ell}=\frac{Y^{T} v}{\sqrt{2} M_{\Sigma}}$, which can easily be obtained from the expressions given in the interactions between Eqs. (13)-(17). The limit on $V_{\ell}$ for the electron flavor in the type-III seesaw scenario is $V_{e}<0.019$ from the electroweak precision data as stated in Refs. $[26,73]$. For the time being, we are considering the $e^{-} e^{+}$and $e^{-} p$ colliders; therefore, we probe $V_{e}$ from a variety of the final states including electron.

Using Eqs. (13)-(17) and the expression for the mixing, we calculate the partial decay widths of the neutral multiplet $\left(\Sigma^{0}\right)$ of the triplet fermion as

$$
\begin{aligned}
\Gamma\left(\Sigma^{0} \rightarrow \ell^{+} W\right) & =\Gamma\left(\Sigma^{0} \rightarrow \ell^{-} W\right) \\
& =\frac{g^{2}\left|V_{\ell}\right|^{2}}{64 \pi}\left(\frac{M_{\Sigma}^{3}}{M_{W}^{2}}\right)\left(1-\frac{M_{W}^{2}}{M_{\Sigma}^{2}}\right)^{2}\left(1+2 \frac{M_{W}^{2}}{M_{\Sigma}^{2}}\right) \\
\Gamma\left(\Sigma^{0} \rightarrow \nu Z\right) & =\frac{g^{2}\left|V_{\ell}\right|^{2}}{64 \pi \cos ^{2} \theta_{W}}\left(\frac{M_{\Sigma}^{3}}{M_{Z}^{2}}\right)\left(1-\frac{M_{Z}^{2}}{M_{\Sigma}^{2}}\right)^{2}\left(1+2 \frac{M_{Z}^{2}}{M_{\Sigma}^{2}}\right) \\
\Gamma\left(\Sigma^{0} \rightarrow \nu h\right) & =\frac{g^{2}\left|V_{\ell}\right|^{2}}{64 \pi}\left(\frac{M_{\Sigma}^{3}}{M_{W}^{2}}\right)\left(1-\frac{M_{h}^{2}}{M_{\Sigma}^{2}}\right)^{2}
\end{aligned}
$$

for the Majorana neutrinos and the partial decay widths of the charged multiplet $\left(\Sigma^{ \pm}\right)$of the triplet fermion as

$$
\begin{aligned}
\Gamma\left(\Sigma^{ \pm} \rightarrow \nu W\right) & =\frac{g^{2}\left|V_{\ell}\right|^{2}}{32 \pi}\left(\frac{M_{\Sigma}^{3}}{M_{W}^{2}}\right)\left(1-\frac{M_{W}^{2}}{M_{\Sigma}^{2}}\right)^{2}\left(1+2 \frac{M_{W}^{2}}{M_{\Sigma}^{2}}\right) \\
\Gamma\left(\Sigma^{ \pm} \rightarrow \ell Z\right) & =\frac{g^{2}\left|V_{\ell}\right|^{2}}{64 \pi \cos ^{2} \theta_{W}}\left(\frac{M_{\Sigma}^{3}}{M_{Z}^{2}}\right)\left(1-\frac{M_{Z}^{2}}{M_{\Sigma}^{2}}\right)^{2}\left(1+2 \frac{M_{Z}^{2}}{M_{\Sigma}^{2}}\right) \\
\Gamma\left(\Sigma^{ \pm} \rightarrow \ell h\right) & =\frac{g^{2}\left|V_{\ell}\right|^{2}}{64 \pi}\left(\frac{M_{\Sigma}^{3}}{M_{W}^{2}}\right)\left(1-\frac{M_{h}^{2}}{M_{\Sigma}^{2}}\right)^{2} .
\end{aligned}
$$

$M_{W}, M_{Z}$, and $M_{h}$ are the $W, Z$, and Higgs boson masses, respectively, in the SM. If the mass splitting $(\Delta M)$ between the charged $\left(\Sigma^{ \pm}\right)$and neutral $\left(\Sigma^{0}\right)$ multiplets induced by the SM gauge bosons is of the order of the pion mass [88], $\Sigma^{ \pm}$ can show the additional decay modes

$$
\begin{aligned}
\Gamma\left(\Sigma^{ \pm} \rightarrow \Sigma^{0} \pi^{ \pm}\right) & =\frac{2 G_{F}^{2} V_{u d}^{2} \Delta M^{3} f_{\pi}^{2}}{\pi} \sqrt{1-\frac{m_{\pi}^{2}}{\Delta M^{2}}} \\
\Gamma\left(\Sigma^{ \pm} \rightarrow \Sigma^{0} e \nu_{e}\right) & =\frac{2 G_{F}^{2} \Delta M^{5}}{15 \pi}
\end{aligned}
$$$$
\Gamma\left(\Sigma^{ \pm} \rightarrow \Sigma^{0} \mu \nu_{\mu}\right)=0.12 \Gamma\left(\Sigma^{ \pm} \rightarrow \Sigma^{0} e \nu_{e}\right),
$$

which are independent of the free parameters. The value of the Fermi constant, $G_{F}$, is $1.1663787 \times 10^{-5} \mathrm{GeV}^{-2}$, the CKM matrix element $V_{u d}$ is $0.97420 \pm 0.00021$, and the $\pi$ meson decay constant, $f_{\pi}$, can be taken as $0.13 \mathrm{GeV}$ from Ref. [89]. The branching ratios (BRs) of $\Sigma^{0}$ and $\Sigma^{ \pm}$into the SM particles are shown in left panel and right panel of Fig. 1 as a function of $M_{\Sigma}$ for $V_{e}=0.019, V_{\mu}=0$, and $V_{\tau}=0$. The BRs of $\Sigma^{0}$ and $\Sigma^{ \pm}$into the SM particles are shown in the left panel and right panel of Fig. 2 as a function of $M_{\Sigma}$ for $V_{e}=V_{\mu}=0.0001$ and $V_{\tau}=0$. In this paper, for further analyses, we consider the case with $V_{e}=0.019, V_{\mu}=0$, and $V_{\tau}=0$ to generate the events and finally to estimate the bounds on the $\left|V_{e}\right|^{2}$.

\section{PRODUCTION CROSS SECTION AND DECAY MODES OF THE $S U(2)_{L}$ TRIPLETS}

The production of the triplet fermion at the $e^{-} e^{+}$and $e^{-} p$ colliders will be followed by its interactions with the SM gauge bosons as described in the Sec. II. At the $e^{-} e^{+}$ collider, the triplet fermion can be produced from the $s$ - and $t$-channel processes being mediated by the photon $(\gamma), W$ boson, and $Z$ boson. The corresponding production modes are given in Fig. 3. The associate production of $\Sigma^{0}$ and 

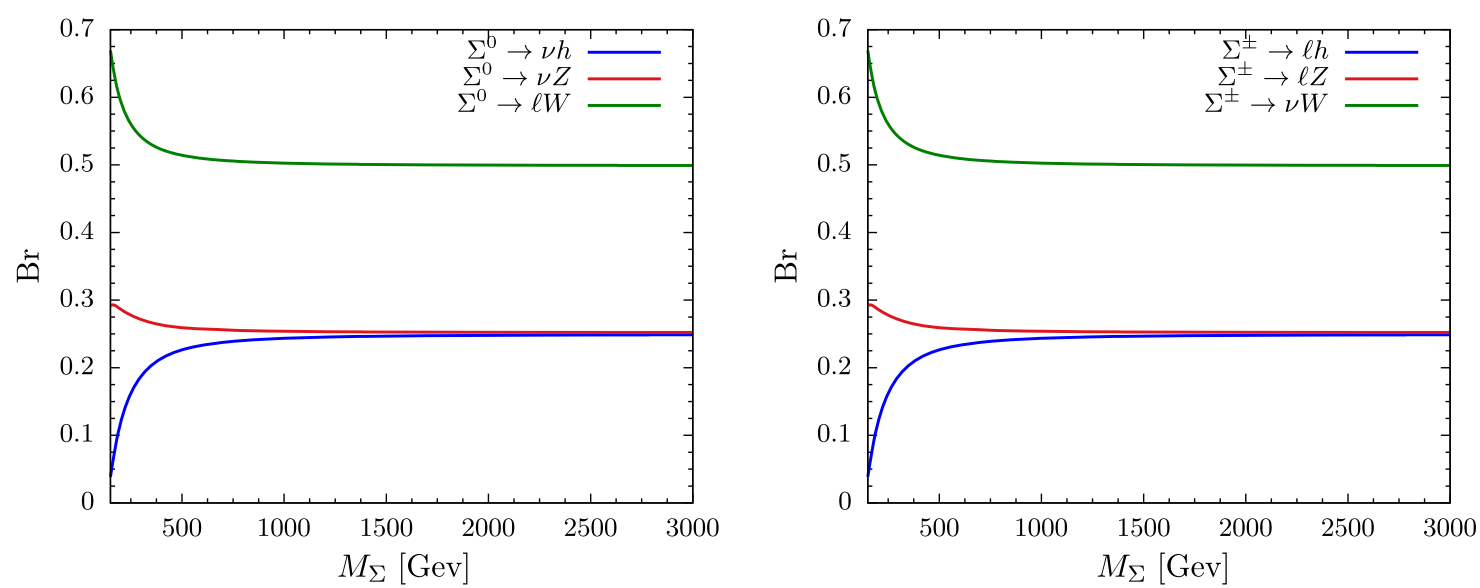

FIG. 1. Branching ratio (Br) of $\Sigma^{0}$ (left) and $\Sigma^{ \pm}$(right) into the SM particles as a function of $M_{\Sigma}$ for $V_{e}=0.019, V_{\mu}=0$, and $V_{\tau}=0$.
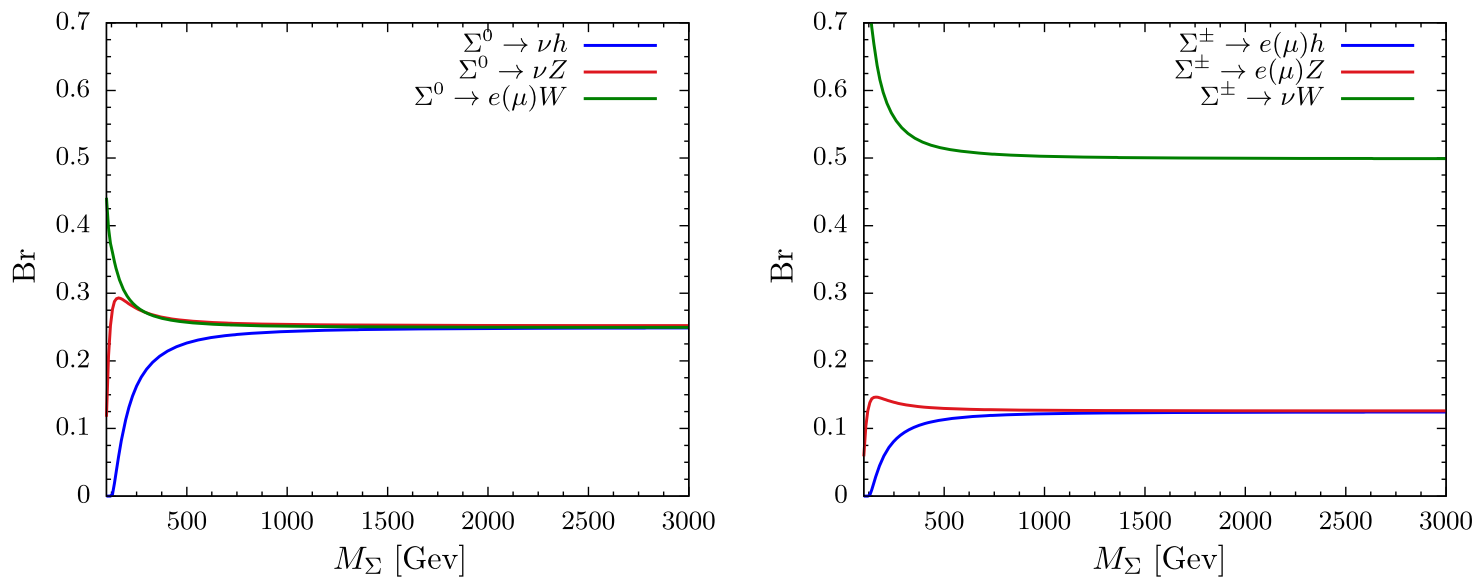

FIG. 2. Branching ratio (Br) of $\Sigma^{0}$ (left) and $\Sigma^{ \pm}$(right) into the SM particles as a function of $M_{\Sigma}$ for $V_{e}=V_{\mu}=0.0001$ and $V_{\tau}=0$.

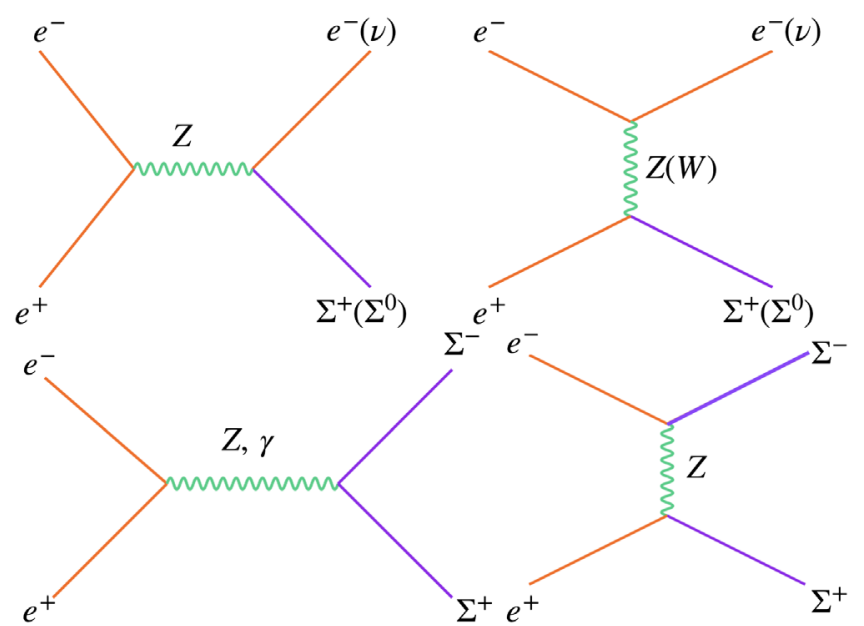

FIG. 3. The production modes of the $\Sigma^{0}$ and $\Sigma^{ \pm}$at the $e^{-} e^{+}$ collider.
$\Sigma^{+}\left(\Sigma^{-}\right)$with the SM leptons (electron and neutrino) are suppressed by the light-heavy mixing square $\left(\left|V_{e}\right|^{2}\right)$. These production modes have been shown in the upper panel of the Fig. 3. At the $e^{+} e^{-}$collider, there is another interesting production channel where $\Sigma^{ \pm}$is produced in pair from the $Z$ and $\gamma$ mediated processes. Such a pair-production process is direct, i.e., not suppressed by the light-heavy mixing. The corresponding production mode is given in the lower panel of Fig. 3.

The cross sections of different production modes of the triplet fermion at the $e^{-} e^{+}$collider are shown in Fig. 4. In the upper panel of Fig. 4 we show the production cross sections of the triplet for fixed $M_{\Sigma}$ but varying center-ofmass energy from $500 \mathrm{GeV}$ to $3 \mathrm{TeV}$. In this case, we fix the triplet mass at $500 \mathrm{GeV}$ (upper, left) and $1 \mathrm{TeV}$ (upper, right), respectively. In the lower panel, we show the production cross section as a function of $M_{\Sigma}$, fixing the center-of-mass energy at 1 (lower, left) and $3 \mathrm{TeV}$ (lower, right), respectively. The triplet fermion can be produced at 

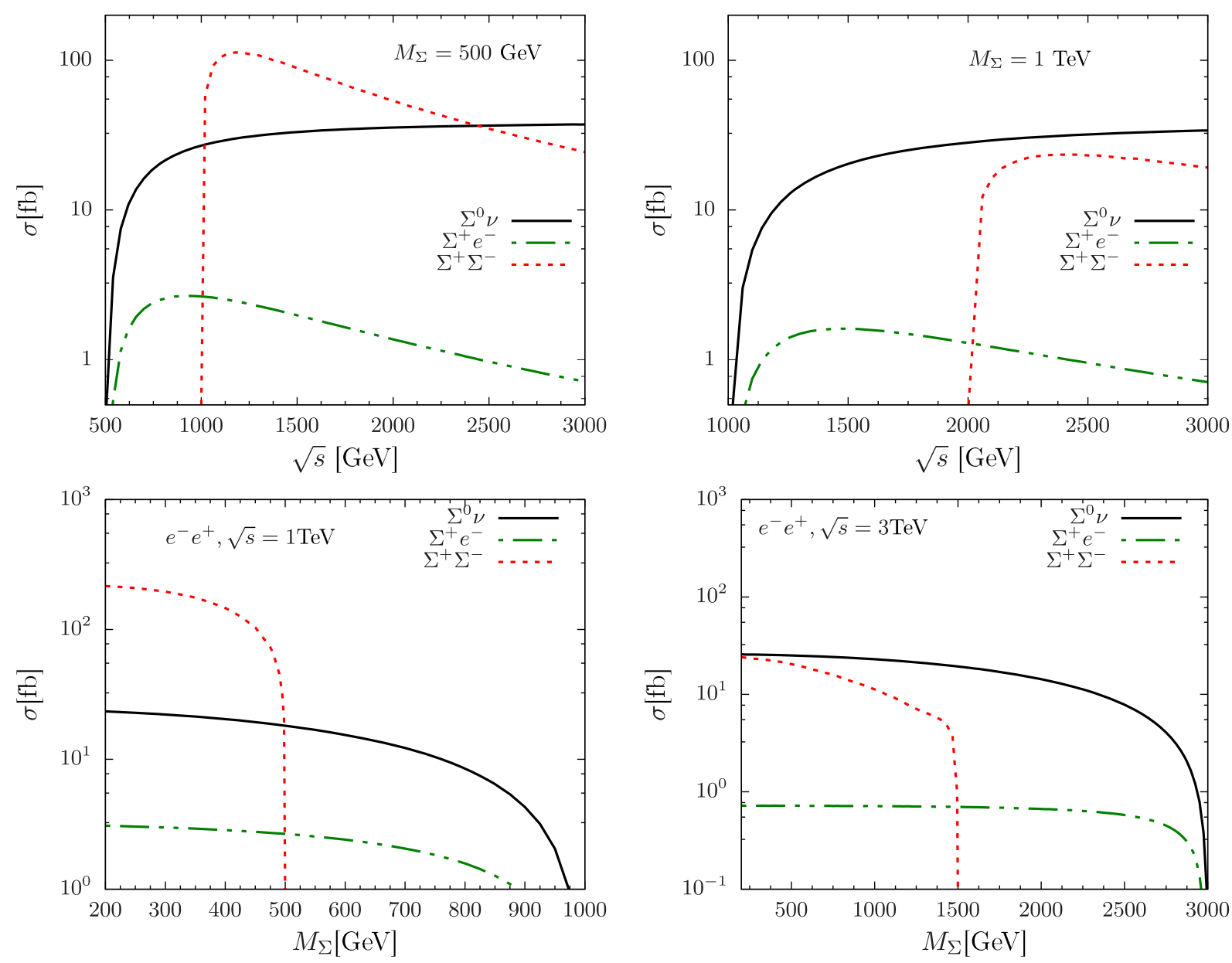

FIG. 4. Cross section for the triplet fermion production at the $e^{-} e^{+}$collider. The production cross sections for the varying center-ofmass energy $(\sqrt{s})$ are shown in the upper panel, fixing the mass of the triplet at $M_{\Sigma}=500 \mathrm{GeV}$ (upper, left) and at $M_{\Sigma}=1 \mathrm{TeV}$ (upper, right). The production cross sections of the triplet fermion as a function of mass are shown in the lower panel for two different values of $\sqrt{s}$ at $1 \mathrm{TeV}$ (lower, left) and $3 \mathrm{TeV}$ (lower, right). To calculate the associate production of the $\Sigma^{ \pm}, \Sigma^{0}$ with the SM leptons, we consider $V_{e}=0.019[26,73]$; however, the pair production of $\Sigma^{+} \Sigma^{-}$is direct.

the $e^{-} p$ collider in association with a jet through the $t$-channel process exchanging the $W$ and $Z$ bosons. The production process is shown in Fig. 5. We consider this process at $1.3 \mathrm{TeV}, 1.8 \mathrm{TeV}$ and $3.46 \mathrm{TeV}$ center of mass energies. At the $e^{-} p$ collider, the electron beam energy has been fixed at $60 \mathrm{GeV}$, but proton beam energies are 7, 13.5, and $50 \mathrm{TeV}$, respectively. The production processes of the $\Sigma^{0} j$ and $\Sigma^{-} j$ are suppressed by the corresponding lightheavy mixing. The production cross sections are shown in Fig. 6 as a function of the $M_{\Sigma}$ for fixed center-of-mass energy $(\sqrt{s})$.

After the production of $\Sigma^{0}$ and $\Sigma^{ \pm}$at the $e^{-} e^{+}$and $e^{-} p$ colliders, the particles will decay into the respective modes according to Eqs. (19) and (20). In this article, we consider the heavier triplet fermion so that it can sufficiently boost the decay products to form a fat jet; hence, we study the signal and the SM backgrounds.

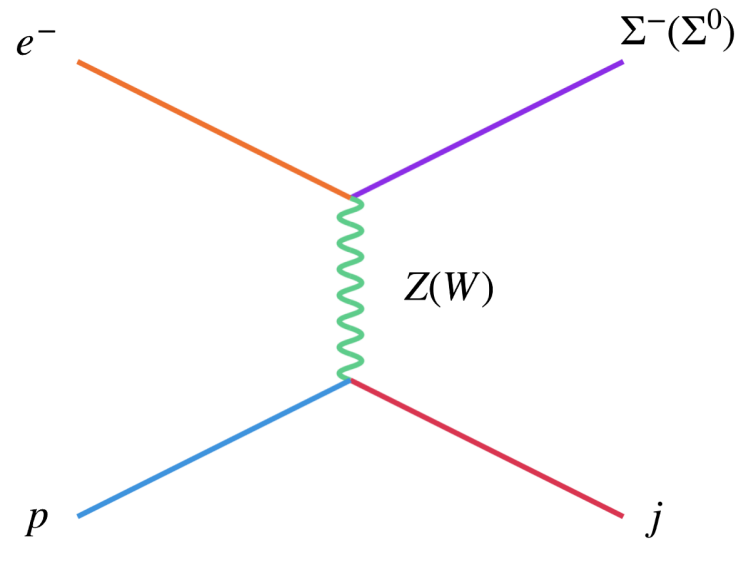

FIG. 5. The production modes of the $\Sigma^{0}$ and $\Sigma^{-}$at the $e^{-} p$ collider, which is suppressed by $\left|V_{\ell}\right|^{2}$. 

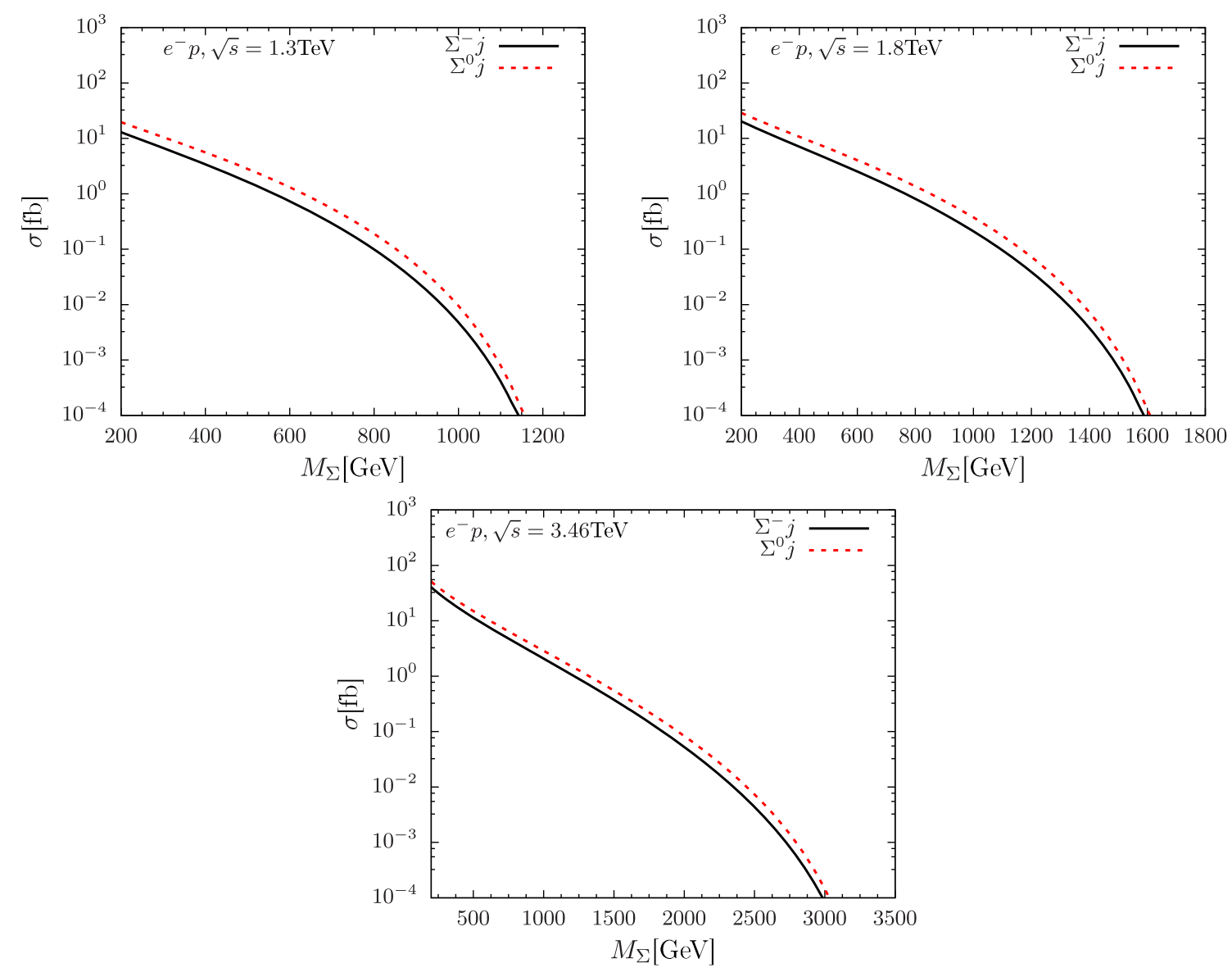

FIG. 6. Cross section for the processes, $\Sigma^{0} j$ and $\Sigma^{-} j$, in the $e^{-} p$ collider as a function of $M_{\Sigma}$ with fixed $\sqrt{s}$ at 1.3 (top, left), 1.8 (top, right), and $3.46 \mathrm{TeV}$ (bottom). We have considered $V_{e}=0.019[26,73]$ to calculate the production cross sections.

\section{COLLIDER ANALYSIS}

To find the discovery prospect, we first implement the model in FeynRules [90] framework, generated signal, and the SM backgrounds using the Monte Carlo event generator MADGRAPH5-aMCNLO [91]. For the subsequent decay, initial-state radiation, final-state radiation, and hadronization, we have used PYTHIA6 [92] for $e^{-} p$ and PYTHIA8 [93] for $e^{-} e^{+}$colliders. We have considered the high-mass regime of the triplet so that the daughter particles from triplet can be sufficiently boosted. Because of the large mass gap between triplet fermion and the SM gauge bosons $(W, Z)$ and SM Higgs $(h)$, the hadronic decay modes of $W$, $Z$, and $h$ can be collimated so that we will have a single jet called a fat jet $(J)$. The fat jet topology is a very powerful tool to significantly reduce the SM backgrounds. We perform the detector simulation using DELPHES3.4.1 [94]. The detector card for the $e^{-} p$ collider has been obtained from Ref. [95]. We use the ILD card (ILC detector card) for the $e^{-} e^{+}$collider in DELPHES. In our analysis, the jets are reconstructed by Cambridge-Achen algorithm [96,97] implemented in the FastJet $[98,99]$ package with the radius parameter as $R=0.8$. We study the production of the triplet fermion and its subsequent decay modes at the $e^{-} e^{+}$ and $e^{-} p$ colliders, respectively. We consider two scenarios at $e^{-} e^{+}$collider in which the center-of-mass energies are $\sqrt{s}=1$ and $3 \mathrm{TeV}$. For $e^{-} p$ collider, we consider the case in which $\sqrt{s}=3.46 \mathrm{TeV}$. In this case, the electron and proton beam energies are $60 \mathrm{GeV}$ and $50 \mathrm{TeV}$, respectively.

At the $e^{-} e^{+}$collider, the following set of signals after the production of the triplet fermion can be found:

(1) $e^{-} e^{+} \rightarrow \Sigma^{0} \nu\left(\Sigma^{ \pm} e^{\mp}\right), \quad \Sigma^{0} \rightarrow e^{\mp} W^{\mp}\left(\Sigma^{ \pm} \rightarrow \nu W^{ \pm}\right)$, and $W^{\mp} \rightarrow J$, where $J$ is the fat jet coming from boosted $W$ boson. The corresponding Feynman diagram is shown in Fig. 7.

(2) $e^{-} e^{+} \rightarrow \Sigma^{0} \nu\left(\Sigma^{ \pm} e^{\mp}\right), \quad \Sigma^{0} \rightarrow h \nu\left(\Sigma^{ \pm} \rightarrow e^{ \pm} h\right), \quad$ and $h \rightarrow J_{b}$, where $J_{b}$ is the fat $b$ jet coming from the boosted SM Higgs decay. The corresponding Feynman diagram is shown in Fig. 13.

(3) $e^{-} e^{+} \rightarrow \Sigma^{+} e^{-}, \Sigma^{+} \rightarrow e^{+} Z$, and $Z \rightarrow J$. The corresponding Feynman diagram is shown in Fig. 15.

(4) $e^{-} e^{+} \rightarrow \Sigma^{+} \Sigma^{-}$, and $\Sigma^{ \pm} \rightarrow W^{ \pm} \nu$, and $W^{ \pm} \rightarrow J$. The corresponding Feynman diagram is shown in Fig. 17.

At the $e^{-} p$ collider, we study the signal $e^{-} p \rightarrow \Sigma^{0} j\left(\Sigma^{-} j\right)$ followed by $\Sigma^{0} \rightarrow e^{ \pm} W^{\mp}, W^{\mp} \rightarrow J\left(\Sigma^{0} \rightarrow \nu W^{-}, W^{-} \rightarrow J\right)$. The corresponding Feynman diagram is shown in Fig. 19. 


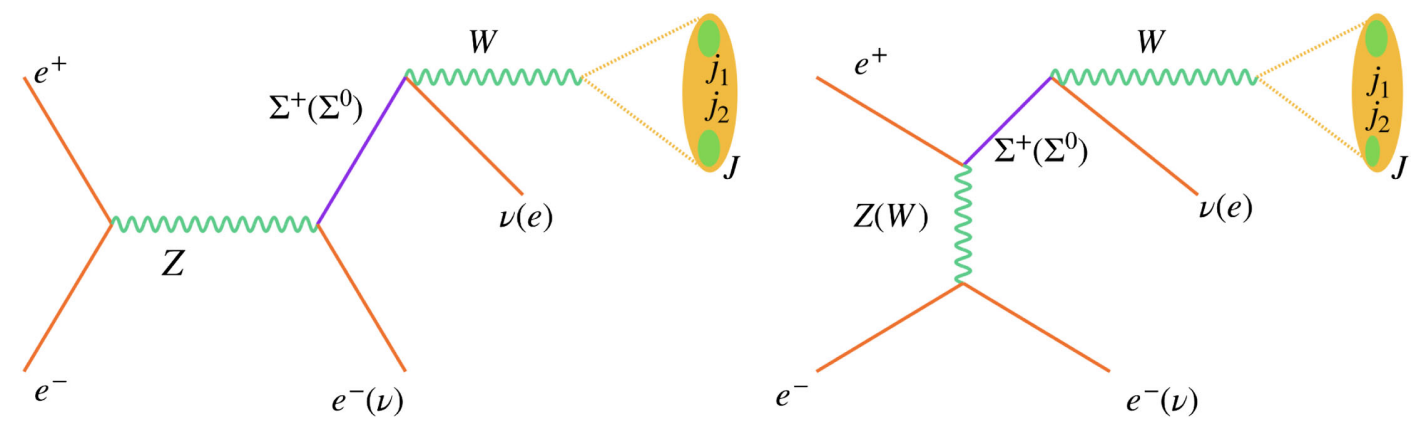

FIG. 7. Fat jet $(J)$ production from the $\Sigma^{+}$and $\Sigma^{0}$ at the $e^{+} e^{-}$collider from the $s$-channel (left) and $t$-channel (right) processes.

As the production cross section for $\Sigma^{0} j$ at the $e p$ collider quickly decreases with increasing triplet mass, we decide to study the signal coming from the dominant decay mode $\Sigma^{0} \rightarrow e W$.

For the analyses of the signal and background events, we use the following set of basic cuts:

(1) Electrons in the final state should have the following transverse momentum $\left(p_{T}^{e}\right)$ and pseudorapidity $\left(\left|\eta^{e}\right|\right): p_{T}^{e}>10 \mathrm{GeV},\left|\eta^{e}\right|<2.5$ (for $e p$ collider, $\left.\left|\eta^{e}\right|<5\right)$.

(2) Jets are ordered in $p_{T}$, and they should have $p_{T}^{j}>$ $10 \mathrm{GeV}$ and $\left|\eta^{j}\right|<2.5$ (for $e^{-} p$ collider, $\left|\eta^{j}\right|<5$ ).

(3) Leptons should be separated by $\Delta R_{\ell \ell}>0.2$.

(4) The jets and leptons should be separated by $\Delta R_{\ell j}>0.3$.

(5) The fat jet is constructed with radius parameter $R=0.8$.

\section{A. Analysis for the final state $e^{ \pm}+J+p_{T}^{\text {miss }}$ at $\sqrt{s}=1$ and $3 \mathrm{TeV} e^{-} e^{+}$colliders}

The final state $e^{ \pm}+J+p_{T}^{\text {miss }}$ arises from the production of $e^{-} e^{+} \rightarrow \Sigma^{0} \nu$ and the subsequent decay of $\Sigma^{0}$ to its dominant channel $e^{ \pm} W^{\mp}$ at the $e^{-} e^{+}$collider. The corresponding Feynman diagram is given in Fig. 7. The $W$ boson can further decay to pair of jets. As we are considering the heavy mass region of the triplet fermion, the $W$ boson will be boosted, and its hadronic decay products, jets, will be collimated such that they can form a fat jet $(J)$.

There are a number of SM processes like $\nu_{e} e W, W W$, $Z Z$, and $t \bar{t}$ which can mimic this final state which are the significant SM backgrounds. Among these channels, $\nu_{e} e W$ and $W W$ give the dominant contributions. We have shown the normalized distributions of missing momentum, $\left|\cos \theta_{e}\right|$, fat jet $p_{T}$, leading lepton $p_{T}$, and fat jet invariant mass distributions in Figs. 8-12 for the signal from $e^{-} e^{+} \rightarrow$ $\Sigma^{0} \nu$ and the SM backgrounds. For these distributions, we have chosen the benchmark points $M_{\Sigma}=900 \mathrm{GeV}$ at $\sqrt{s}=1 \mathrm{TeV}$ and $M_{\Sigma}=1,2 \mathrm{TeV}$ at $\sqrt{s}=3 \mathrm{TeV}$. Note that for the case of the SM backgrounds the invariant mass distribution of the fat jet $\left(m_{J}\right)$ has also low-energy peaks $\left(m_{J} \leq 25 \mathrm{GeV}\right)$ which come from the hadronic activity of the low-energy jets. Hence, a high $m_{J}$ cut will be useful to reduce SM backgrounds.

Because of the heavy mass of the triplet fermion, the leading lepton and the fat jet $p_{T}$ distributions for the signal will be in the high values than the SM backgrounds. Hence,

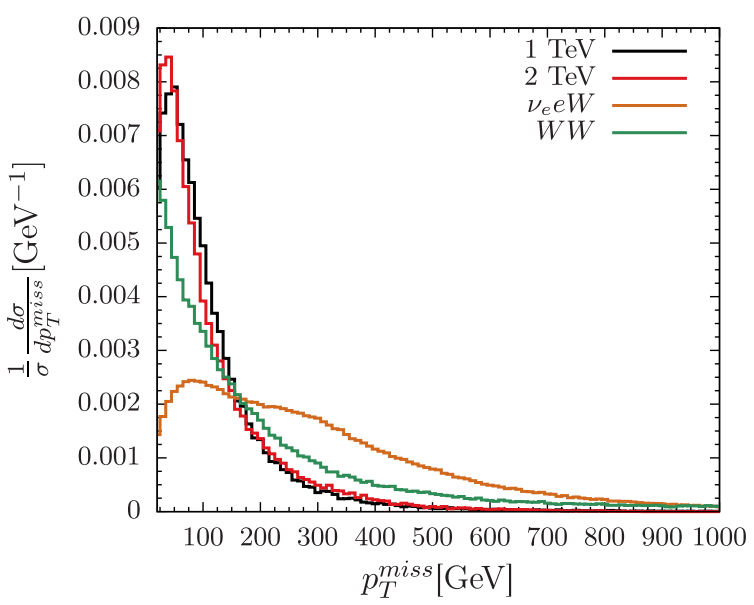

FIG. 8. Normalized missing momentum distributions of the signal and background events for $M_{\Sigma}=900 \mathrm{GeV}$ at the $\sqrt{s}=1 \mathrm{TeV}$ (left panel) and $M_{\Sigma}=1,2 \mathrm{TeV}$ at the $\sqrt{s}=3 \mathrm{TeV}$ (right panel) at $e^{-} e^{+}$colliders. 

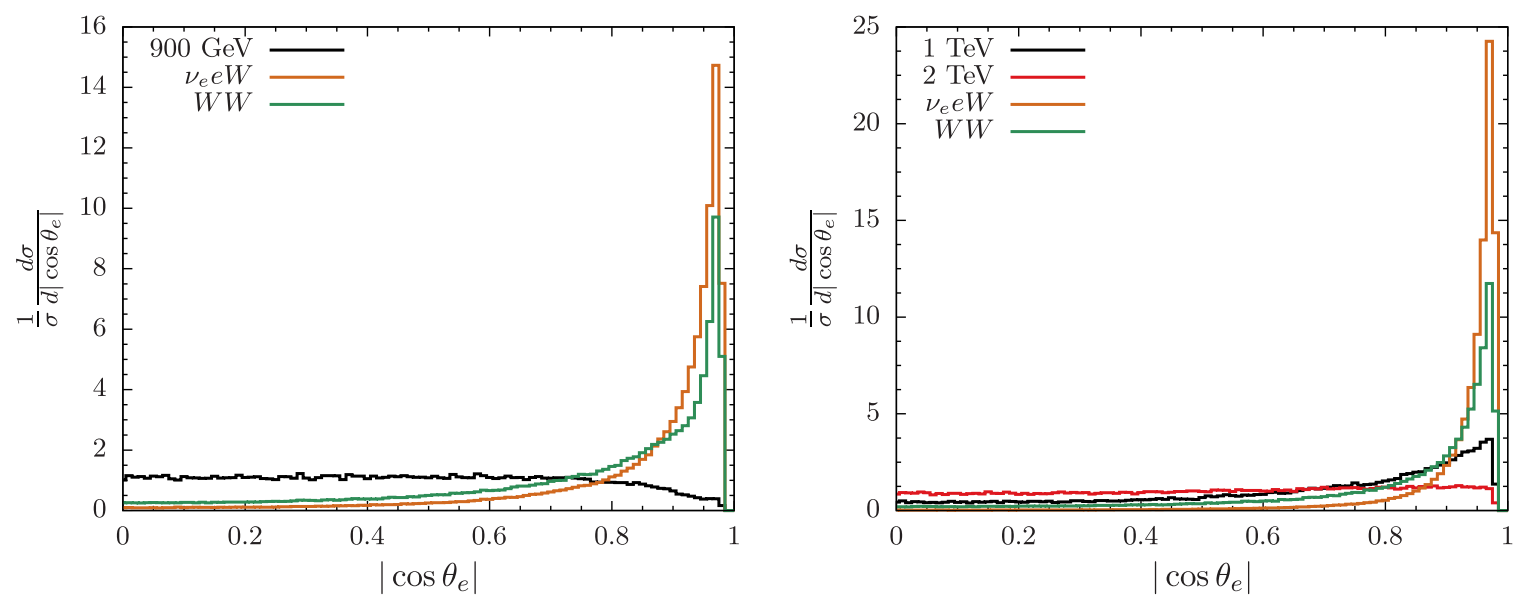

FIG. 9. Same as in Fig. 8, but now for $\cos \theta_{e}$ distribution.
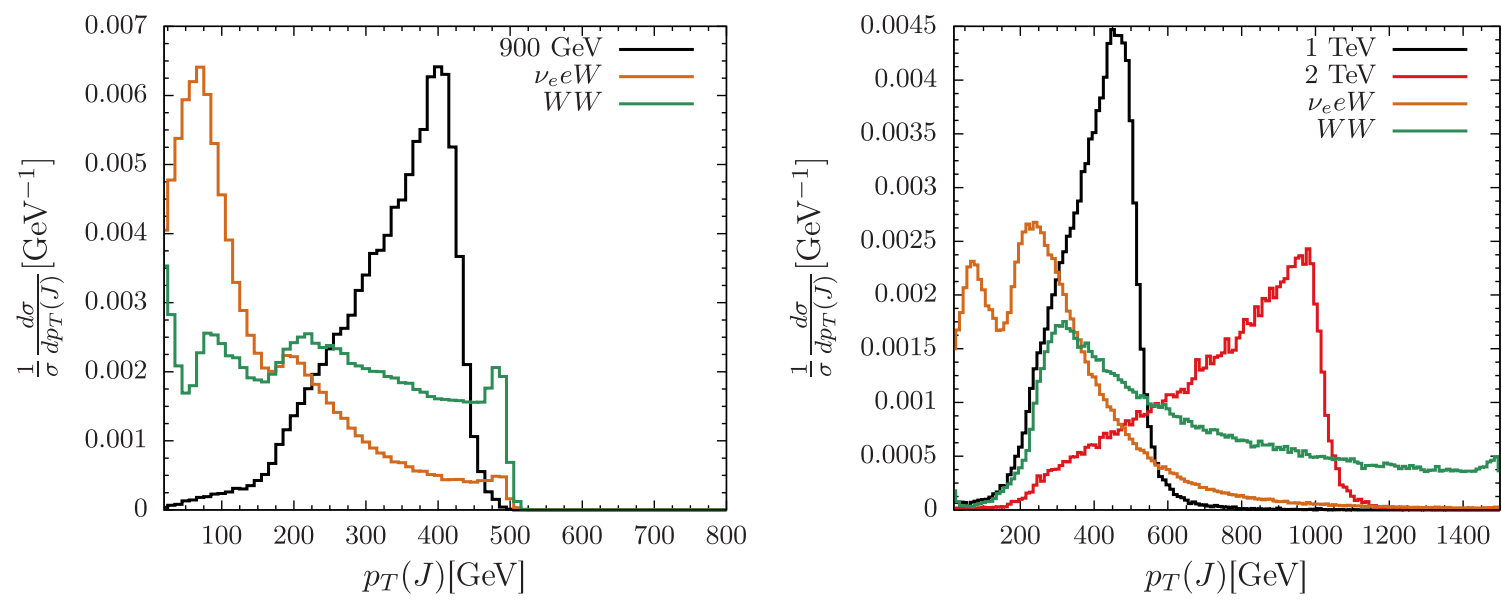

FIG. 10. Same as in Fig. 8, but now for fat jet $p_{T}$ distribution.
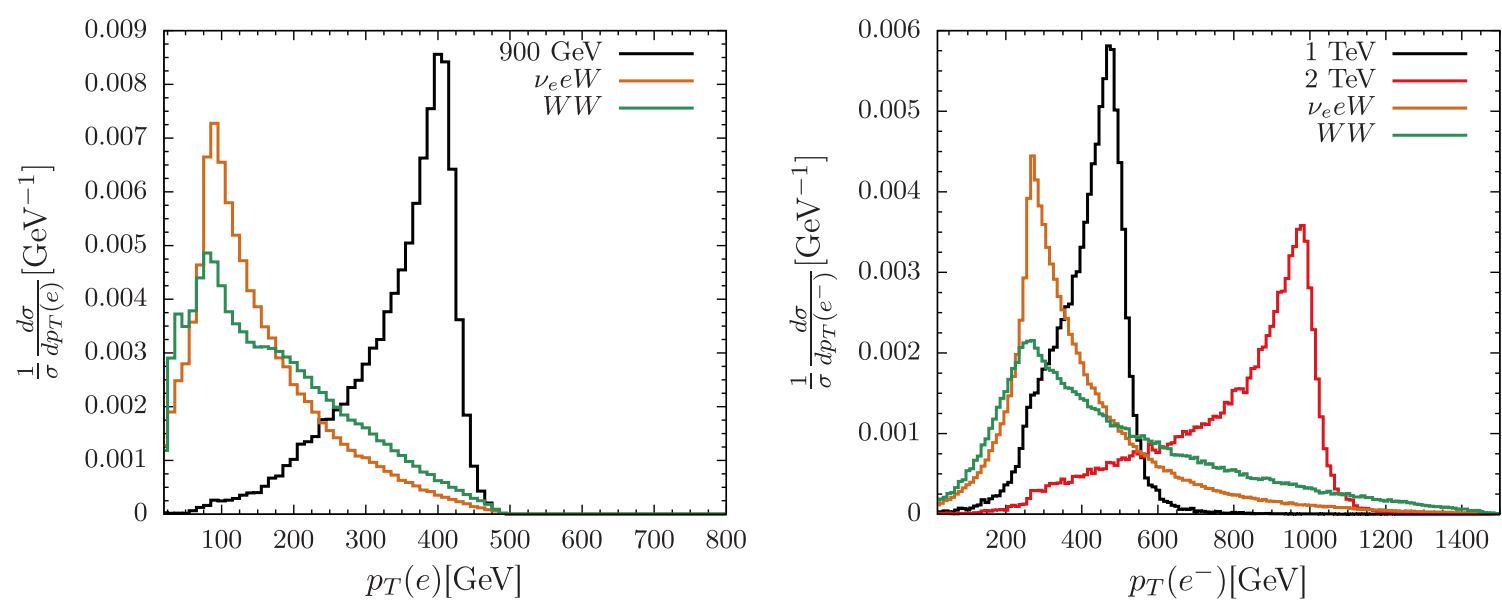

FIG. 11. Same as in Fig. 8, but now for leading lepton $p_{T}$ distribution. 

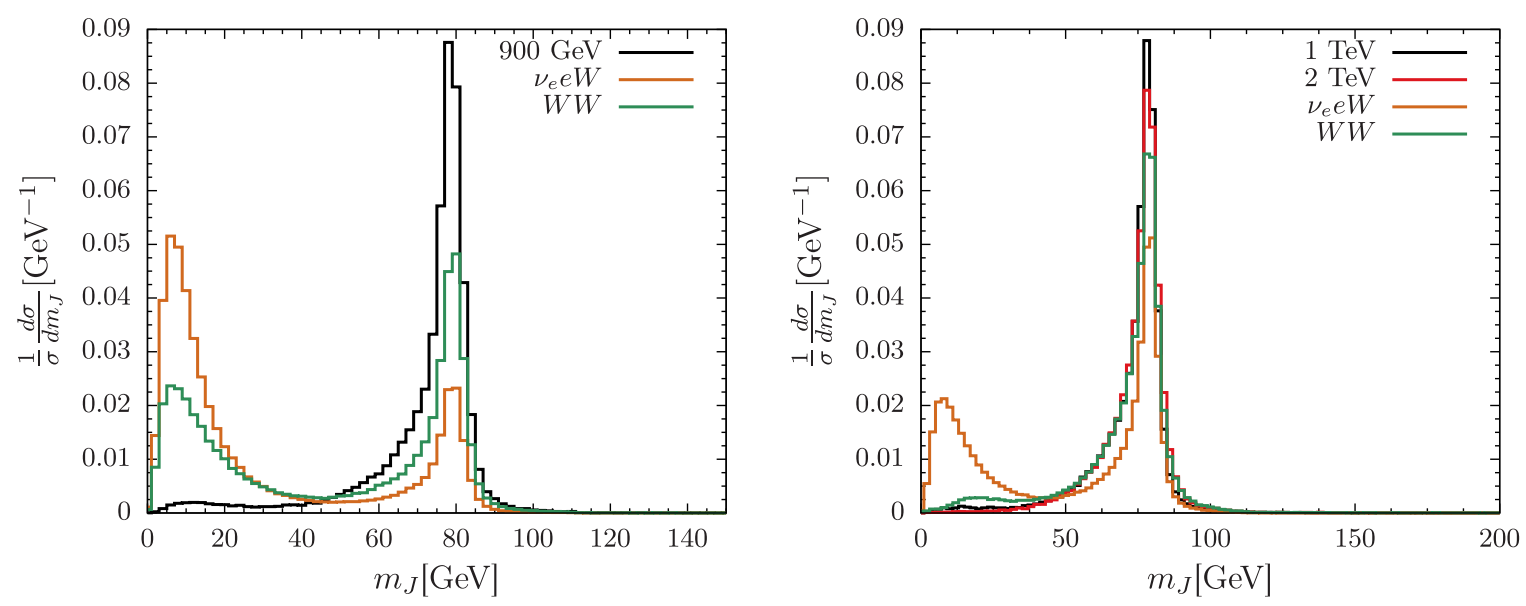

FIG. 12. Same as in Fig. 8, but now for invariant mass distribution of fat jet.

the high $p_{T}$ cut for leading lepton and fat jet will be effective to reduce SM background. At this point, we would like to mention that the same final state can also be obtained from $e^{-} e^{+} \rightarrow \Sigma^{ \pm} e^{\mp}$. We have found that the $p_{T}$ distribution for the electron is mostly in the low-momentum range for this channel. The application of the high $p_{T}$ cut for the electron applied in the $\Sigma^{0} \nu$ channel will significantly cut this channel out so that its contribution becomes negligible. The high $p_{T}$ cut for the electron from the $\Sigma^{0} \nu$ process is required because the electron in this process is coming from the heavy triplet in contrary to the $\Sigma^{ \pm} e^{\mp}$ process. Hence, in the further analyses, we neglect events from the $\Sigma^{ \pm} e^{\mp}$ process.

The polar angle variable for the electron $\cos \theta_{e}$ in Fig. 9 is defined as $\theta_{e}=\tan ^{-1}\left(\frac{p_{T}^{e}}{p_{z}^{e}}\right)$, where $p_{z}^{e}$ is the $z$ component of the 3-momentum of the electron. At the $e^{-} e^{+}$collider, the polar angle cut is very effective to reduce the SM backgrounds.

To study this process, we have chosen the triplet mass $M_{\Sigma}=800-950 \mathrm{GeV}$ for $\sqrt{s}=1 \mathrm{TeV}$ and $M_{\Sigma}=$ $800 \mathrm{GeV}-2.9 \mathrm{TeV}$ for $\sqrt{s}=3 \mathrm{TeV}$. In view of the distributions in Figs. 8-12, we have used the following set of advanced selection cuts to reduce the SM backgrounds further:

(i) Advanced cuts for $M_{\Sigma}=800-900 \mathrm{GeV}$ at the $\sqrt{s}=$ $1 \mathrm{TeV} e^{-} e^{+}$collider after the detector simulation:

(1) polar angle of the lepton and the fat jet $\left|\cos \theta_{e}\right|<$ 0.9 ,

(2) transverse momentum for the fat jet $p_{T}^{J}>$ $300 \mathrm{GeV}$,

(3) transverse momentum for the leading lepton $p_{T}^{e^{ \pm}}>300 \mathrm{GeV}$

(4) fat jet mass $m_{J}>70 \mathrm{GeV}$.

(ii) Advanced cuts for $M_{\Sigma}=800 \mathrm{GeV}-2.9 \mathrm{TeV}$ at the $\sqrt{s}=3 \mathrm{TeV} e^{-} e^{+}$collider after the detector simulation:

(1) polar angle of the lepton and the fat jet $\left|\cos \theta_{e}\right|<0.9$,
(2) transverse momentum for the fat jet $p_{T}^{J}>$ $200 \mathrm{GeV}$ for $800 \mathrm{GeV} \leq M_{\Sigma} \leq 1.5 \mathrm{TeV}$ and $p_{T}^{J}>500 \mathrm{GeV}$ for $1.6 \mathrm{TeV} \leq M_{\Sigma} \leq 2.9 \mathrm{TeV}$,

(3) transverse momentum for the leading lepton $p_{T}^{e^{ \pm}}>200 \mathrm{GeV}$ for $800 \mathrm{GeV} \leq M_{\Sigma} \leq 1.5 \mathrm{TeV}$ and $p_{T}^{e^{ \pm}}>500 \mathrm{GeV}$ for $1.6 \mathrm{TeV} \leq M_{\Sigma} \leq 2.9 \mathrm{TeV}$,

(4) fat jet mass $m_{J}>70 \mathrm{GeV}$.

We have shown the cut flow for $M_{\Sigma}=900 \mathrm{GeV}$ (at $\sqrt{s}=1 \mathrm{TeV}$ ) and $M_{\Sigma}=1,2 \mathrm{TeV}$ (at $\sqrt{s}=3 \mathrm{TeV}$ ) in Tables I-III. Note that setting the important variable $\cos \theta_{e}$ as $\left|\cos \theta_{e}\right| \leq 0.9$ gives a very strong cut for the $\mathrm{SM}$ backgrounds. To study the heavier triplet fermion at the $\sqrt{s}=3 \mathrm{TeV} e^{-} e^{+}$collider, we have chosen stronger cuts for the transverse momenta of the lepton and fat jet to reduce the SM backgrounds.

\section{B. Analysis for the final state $J_{b}+p_{T}^{\text {miss }}$ at $\sqrt{s}=3 \mathrm{TeV} e^{-} e^{+}$collider}

This final state $J_{b}+p_{T}^{\text {miss }}$ arises from the production of $e^{-} e^{+} \rightarrow \Sigma^{0} \nu$ (conjugate process implied) and the subsequent decay $\Sigma^{0}$ to $h \nu$ at the $e^{-} e^{+}$collider. The corresponding Feynman diagram is given in Fig. 13. The SM Higgs $(h)$ branching ratio is approximately $60 \%$ to $b \bar{b}$ for $m_{h}=125 \mathrm{GeV}$, which is the reason for our consideration of this channel. As $h$ is boosted in our case, we will have a collimated fat- $b$ jet. For this final state, the dominant SM backgrounds come from the process $h \nu_{\ell} \overline{\nu_{\ell}}$ and $Z \nu_{\ell} \bar{\nu}_{\ell}$. Backgrounds can also come from the processes like $Z h$ and $Z Z$, with subsequent decays of the $Z$ boson into the light neutrinos and $h \rightarrow b \bar{b}$. We have combined all the SM backgrounds at the time of the event generation. In this work, we consider a flat $70 \%$ tagging efficiency for each of the daughter $b$ jets coming from the Higgs decay.

We have shown the normalized distributions for missing momentum, $p_{T}$ of the fat $b$, and invariant mass $m_{J_{b}}$ in Fig. 14. These distributions are given for $M_{\Sigma}=1$, $2 \mathrm{TeV}$ at the $\sqrt{s}=3 \mathrm{TeV} e^{-} e^{+}$collider along with $\mathrm{SM}$ 
TABLE I. Cut flow for the signal and background cross sections for the final state $e^{ \pm}+J+p_{T}^{\text {miss }}$ for $M_{\Sigma}=$ $900 \mathrm{GeV}$ at the $\sqrt{s}=1 \mathrm{TeV} e^{-} e^{+}$collider.

\begin{tabular}{lcccrrr}
\hline \hline & & \multicolumn{4}{c}{ Background $(\mathrm{fb})$} & \\
\cline { 3 - 6 } Cuts & Signal $(\mathrm{fb})$ & \multicolumn{1}{c}{$\nu_{e} e W$} & $W W$ & \multicolumn{1}{c}{$Z \mathbf{T}$} & $t \bar{t}$ & Total $(\mathrm{fb})$ \\
\hline Basic cuts & 0.898 & 418.647 & 98.415 & 0.476 & 149.562 & 667.101 \\
$\left|\cos \theta_{e}\right| \leq 0.9$ & 0.863 & 165.196 & 58.901 & 0.290 & 149.551 & 373.938 \\
$p_{T}^{J}>300 \mathrm{GeV}$ & 0.679 & 46.136 & 35.567 & 0.073 & 149.418 & 231.194 \\
$p_{T}^{e}>300 \mathrm{GeV}$ & 0.653 & 17.829 & 13.338 & 0.033 & 0.010 & 31.211 \\
$m_{J}>70 \mathrm{GeV}$ & 0.552 & 13.905 & 10.327 & 0.027 & 0.009 & 24.269 \\
\hline \hline
\end{tabular}

TABLE II. Cut flow for the signal and background cross sections for the final state $e^{ \pm}+J+p_{T}^{\text {miss }}$ for $M_{\Sigma}=$ $1 \mathrm{TeV}$ at the $\sqrt{s}=3 \mathrm{TeV} e^{-} e^{+}$collider.

\begin{tabular}{|c|c|c|c|c|c|c|}
\hline \multirow[b]{2}{*}{ Cuts } & \multirow[b]{2}{*}{ Signal (fb) } & \multicolumn{4}{|c|}{ Background (fb) } & \multirow[b]{2}{*}{ Total (fb) } \\
\hline & & $\nu_{e} e W$ & $W W$ & $Z Z$ & $t \bar{t}$ & \\
\hline Basic cuts & 4.611 & 267.680 & 12.686 & 0.138 & 0.182 & 280.687 \\
\hline$\left|\cos \theta_{e}\right| \leq 0.9$ & 3.418 & 49.100 & 6.198 & 0.059 & 0.150 & 55.508 \\
\hline$p_{T}^{J}>200 \mathrm{GeV}$ & 3.318 & 38.553 & 6.165 & 0.057 & 0.146 & 44.921 \\
\hline$p_{T}^{e}>200 \mathrm{GeV}$ & 3.265 & 36.982 & 5.773 & 0.047 & 0.083 & 42.886 \\
\hline$m_{J}>70 \mathrm{GeV}$ & 2.499 & 27.159 & 4.484 & 0.040 & 0.077 & 31.760 \\
\hline
\end{tabular}

TABLE III. Cut flow for the signal and background cross sections for the final state $e^{ \pm}+J+p_{T}^{\text {miss }}$ for $M_{\Sigma}=$ $2 \mathrm{TeV}$ at the $\sqrt{s}=3 \mathrm{TeV} e^{-} e^{+}$collider.

\begin{tabular}{lcrrrrr}
\hline \hline & & \multicolumn{4}{c}{ Background $(\mathrm{fb})$} & \multicolumn{1}{c}{} \\
\cline { 3 - 5 } Cuts & Signal $(\mathrm{fb})$ & \multicolumn{1}{c}{$\nu_{e} e W$} & $W W$ & \multicolumn{1}{c}{ TZ } & $t \bar{t}$ & Total $(\mathrm{fb})$ \\
\hline Basic cuts & 2.751 & 267.680 & 12.686 & 0.138 & 0.182 & 280.687 \\
$\left|\cos \theta_{e}\right| \leq 0.9$ & 2.474 & 49.100 & 6.198 & 0.059 & 0.150 & 55.508 \\
$p_{T}^{J}>500 \mathrm{GeV}$ & 2.250 & 19.555 & 5.948 & 0.053 & 0.131 & 25.687 \\
$p_{T}^{e}>500 \mathrm{GeV}$ & 2.223 & 15.591 & 4.346 & 0.034 & 0.043 & 20.015 \\
$m_{J}>70 \mathrm{GeV}$ & 1.802 & 12.897 & 3.473 & 0.029 & 0.042 & 16.441 \\
\hline \hline
\end{tabular}

backgrounds. The invariant mass distribution of the fat $b$ coming from the $h$ decay peaks around the Higgs mass for the signal. Hence, a cut like $m_{J_{b}}>100 \mathrm{GeV}$ sets a strong constraint on SM backgrounds. Missing momentum and the $p_{T}$ distribution of the fat $b$ for the signal will likely be in the high values compared to the SM backgrounds due to the high mass of the triplet fermion. We have considered the mass range $M_{\Sigma}=800 \mathrm{GeV}-2.9 \mathrm{TeV}$ for $\sqrt{s}=3 \mathrm{TeV}$.

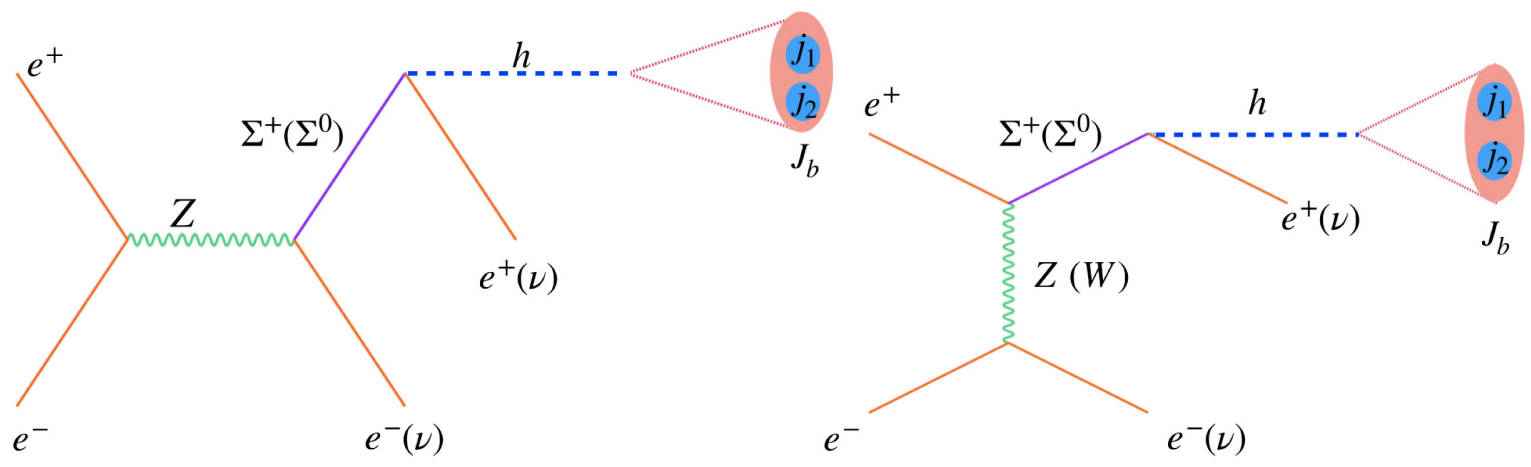

FIG. 13. Fat $b$-jet $\left(J_{b}\right)$ production from the $\Sigma^{+}$and $\Sigma^{0}$ at the $e^{-} e^{+}$collider from the $s$-channel (left) and $t$-channel (right) processes. 

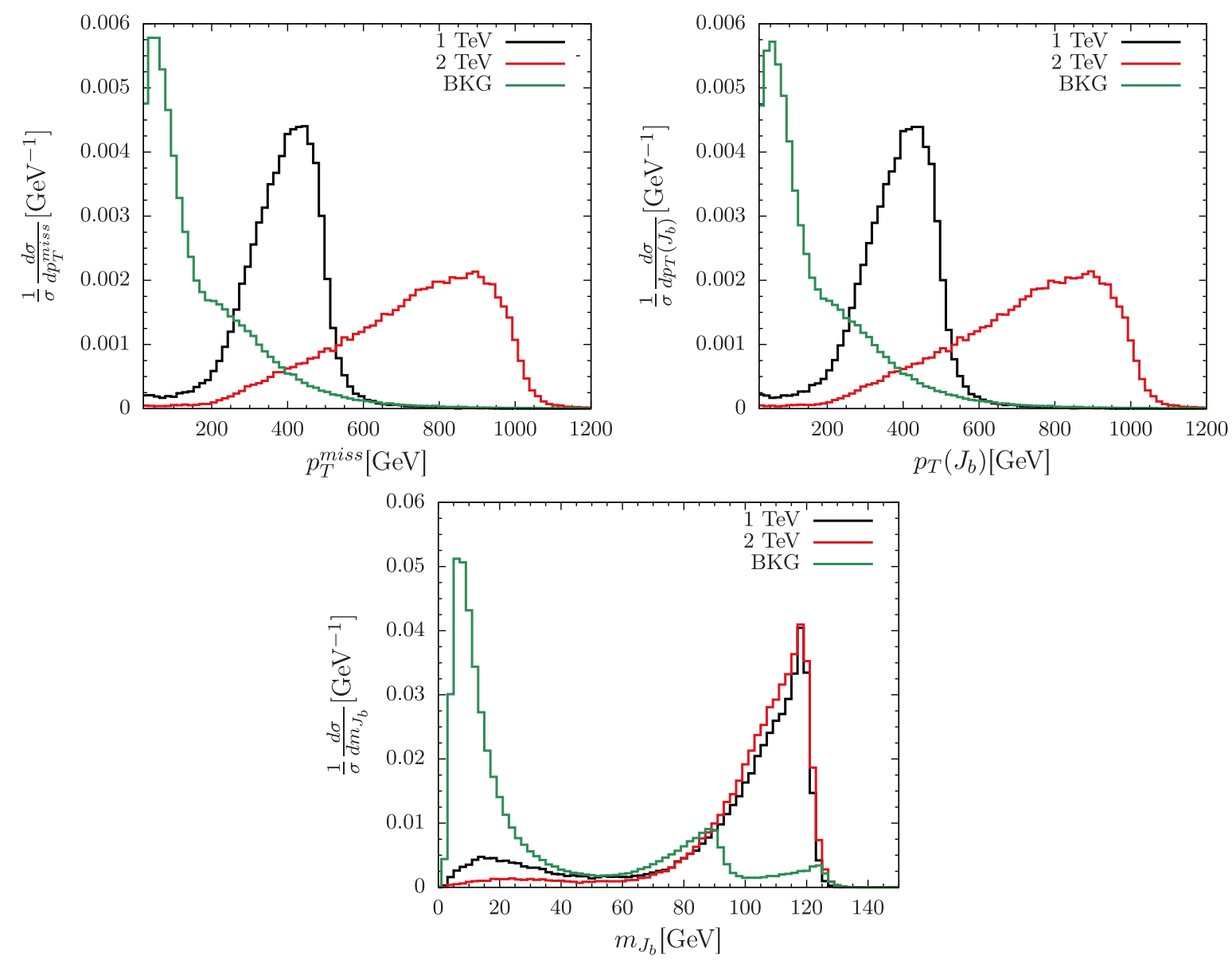

FIG. 14. Normalized distributions of missing momentum, fat- $b p_{T}$ and fat- $b$ invariant mass of the signal and background events for $M_{\Sigma}=1,2 \mathrm{TeV}$ at the $\sqrt{s}=3 \mathrm{TeV}$ at $e^{-} e^{+}$colliders.

In view of these distributions of Fig. 14, we impose the following set of advanced selection cuts to reduce the SM backgrounds further:

(1) missing energy, $p_{T}^{\text {miss }}>300 \mathrm{GeV}$ for $800 \mathrm{GeV} \leq$ $M_{\Sigma} \leq 1.5 \mathrm{TeV}$ and $p_{T}^{\text {miss }}>500 \mathrm{GeV}$ for $1.6 \mathrm{TeV} \leq$ $M_{\Sigma} \leq 2.9 \mathrm{TeV}$

(2) transverse momentum for $J_{b}, p_{T}^{J_{b}}>300 \mathrm{GeV}$ for $800 \mathrm{GeV} \leq M_{\Sigma} \leq 1.5 \mathrm{TeV}$ and $p_{T}^{J_{b}}>500 \mathrm{GeV}$ for $1.6 \mathrm{TeV} \leq M_{\Sigma} \leq 2.9 \mathrm{TeV}$

(3) fat- $b$ mass, $m_{J_{b}}>100 \mathrm{GeV}$.

We have shown the cut flow for two benchmark points $M_{\Sigma}=1$ and $2 \mathrm{TeV}$ at $\sqrt{s}=3 \mathrm{TeV}$ in Tables IV and $\mathrm{V}$, respectively.

TABLE IV. Cut flow for the signal and background cross sections for the final state $J_{b}+p_{T}^{\text {miss }}$ for $M_{\Sigma}=1 \mathrm{TeV}$ at the $\sqrt{s}=3 \mathrm{TeV}$ linear collider.

\begin{tabular}{lcc}
\hline \hline Cuts & Signal $(\mathrm{fb})$ & Background $(\mathrm{fb})$ \\
\hline Basic cuts & 1.203 & 126.225 \\
$p_{T}^{\text {miss }}>300 \mathrm{GeV}$ & 1.007 & 21.664 \\
$p_{T}^{J_{b}}>300 \mathrm{GeV}$ & 1.004 & 21.449 \\
$m_{J_{b}}>100 \mathrm{GeV}$ & 0.713 & 6.470 \\
\hline \hline
\end{tabular}

\section{Analysis for the final state $e^{-} e^{+}+J$ at $\sqrt{s}=3 \mathrm{TeV} e^{-} e^{+}$collider}

In this section, we discuss the potential to test $\Sigma^{+}$in $e^{-} e^{+} \rightarrow e^{-} \Sigma^{+}$mode (conjugate process implied) followed by $\Sigma^{+} \rightarrow e^{+} Z \rightarrow e^{-} e^{+} J$ at the $\sqrt{s}=3 \mathrm{TeV} e^{-} e^{+}$collider. The corresponding Feynman diagram is given in Fig 15. There exist several SM backgrounds for this process. The dominant background arises from $Z / \gamma j j$, whereas, $t \bar{t}$, $W W Z, W W j j$, and $W Z j j$ constitute subdominant contributions. To find the optimized cuts, we first plotted normalized distributions of the leading and subleading electrons and the leading fat jet in Fig. 16 for signal and

TABLE V. Cut flow for the signal and background cross sections for the final state $J_{b}+p_{T}^{\text {miss }}$ for $M_{\Sigma}=2 \mathrm{TeV}$ at the $\sqrt{s}=3 \mathrm{TeV} e^{-} e^{+}$collider.

\begin{tabular}{lcc}
\hline \hline Cuts & Signal $(\mathrm{fb})$ & Background $(\mathrm{fb})$ \\
\hline Basic cuts & 0.887 & 126.225 \\
$p_{T}^{\text {miss }}>500 \mathrm{GeV}$ & 0.757 & 5.025 \\
$p_{T}^{J_{b}}>500 \mathrm{GeV}$ & 0.756 & 5.004 \\
$m_{J_{b}}>100 \mathrm{GeV}$ & 0.571 & 1.501 \\
\hline \hline
\end{tabular}




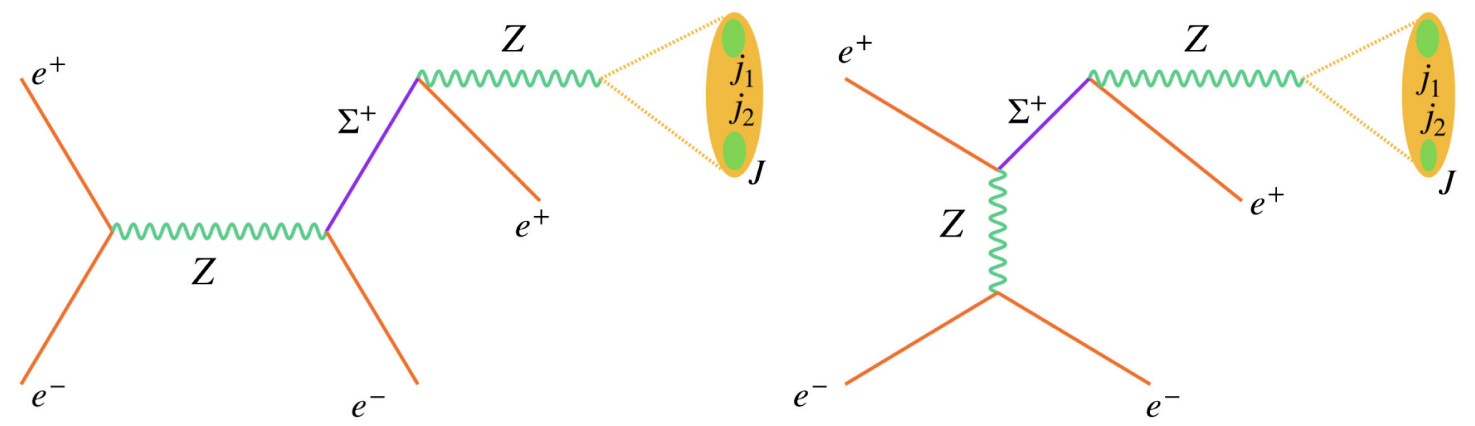

FIG. 15. Fat jet $(J)$ production from the $\Sigma^{+}$at the $e^{-} e^{+}$collider from the $s$-channel (left) and $t$-channel (right) processes.
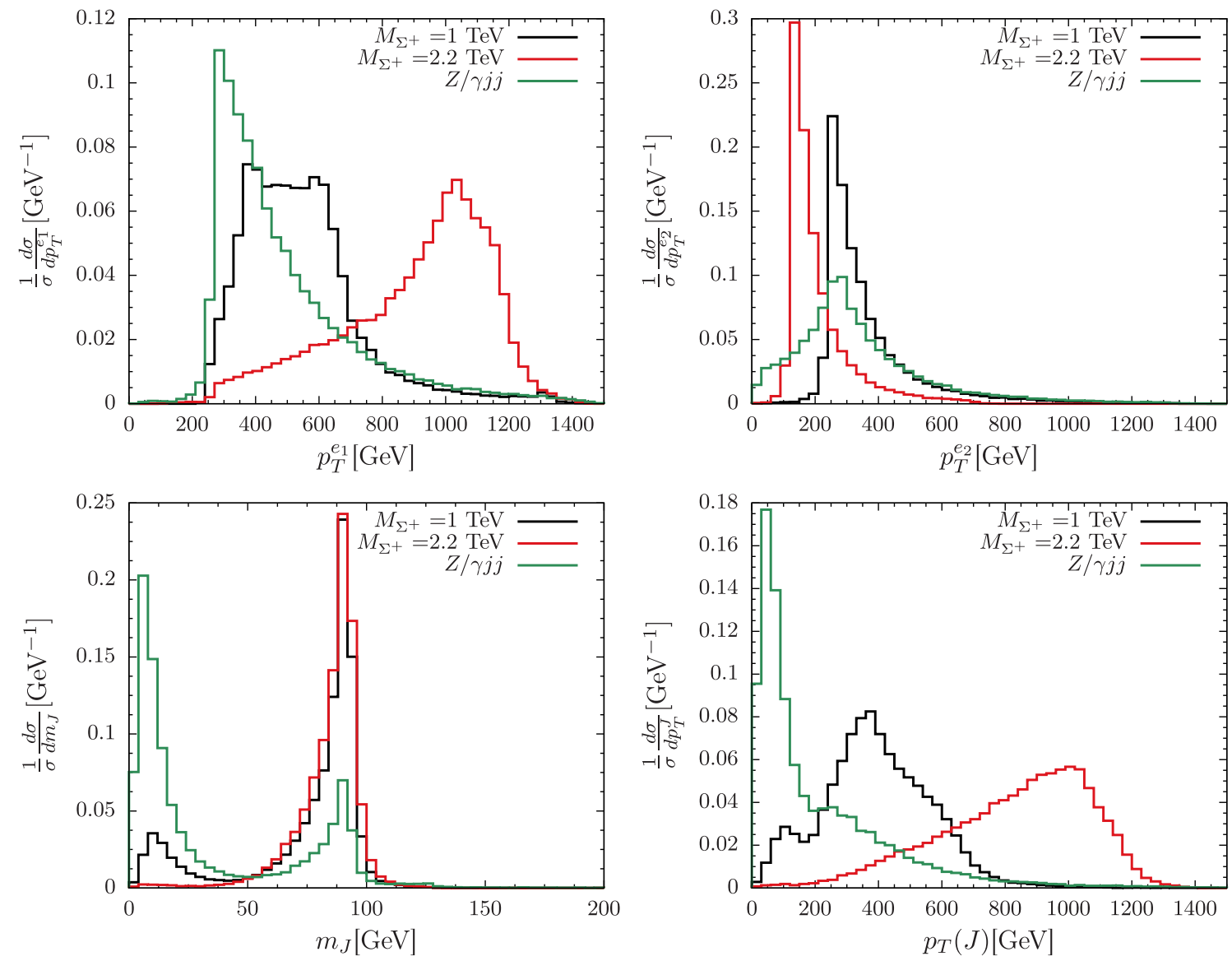

FIG. 16. Normalized $p_{T}^{e_{1}}$ (upper left), $p_{T}^{e_{2}}$ (upper right), $m_{J}$ (lower left), and $p_{T}^{J}$ (lower right) distributions before applying any selection cuts for the $e^{-} e^{+} \rightarrow e^{-} \Sigma^{+} \rightarrow e^{-} e^{+} Z \rightarrow e^{-} e^{+} J$ process. The red and black distributions correspond to signal for $M_{\Sigma}=1 \mathrm{TeV}$ and $M_{\Sigma}=2.2 \mathrm{TeV}$, while the green distribution is for the dominant $Z / \gamma j j$ background.

TABLE VI. Cut flow of the signal and backgrounds for a benchmark $M_{\Sigma}=1 \mathrm{TeV}$ for the signal $e^{-} e^{+} J$ at the $e^{-} e^{+}$collider process.

\begin{tabular}{lccccccc}
\hline \hline Selection cut & Signal $(\mathrm{fb})$ & $Z / \gamma j j(\mathrm{fb})$ & $t \bar{t}(\mathrm{fb})$ & $W W Z(\mathrm{fb})$ & $W W j j(\mathrm{fb})$ & $W Z j j(\mathrm{fb})$ & Total background(fb) \\
\hline Basic cuts & 0.105 & 7.99 & 0.01 & 0.31 & 0.11 & 0.14 & 8.56 \\
$p_{T}^{e_{1}}>350 \mathrm{GeV}, p_{T}^{e_{2}}>200 \mathrm{GeV}$ & 0.092 & 4.59 & 0.001 & 0.06 & 0.02 & 0.03 & 4.7 \\
$p_{T}^{J}>150 \mathrm{GeV}$ & 0.085 & 1.82 & 0.0002 & 0.04 & 0.02 & 0.02 & 1.9 \\
$80 \mathrm{GeV}<m_{J}<100 \mathrm{GeV}$ & 0.057 & 0.8 & 0.00002 & 0.02 & 0.01 & 0.01 & 0.84 \\
\hline \hline
\end{tabular}



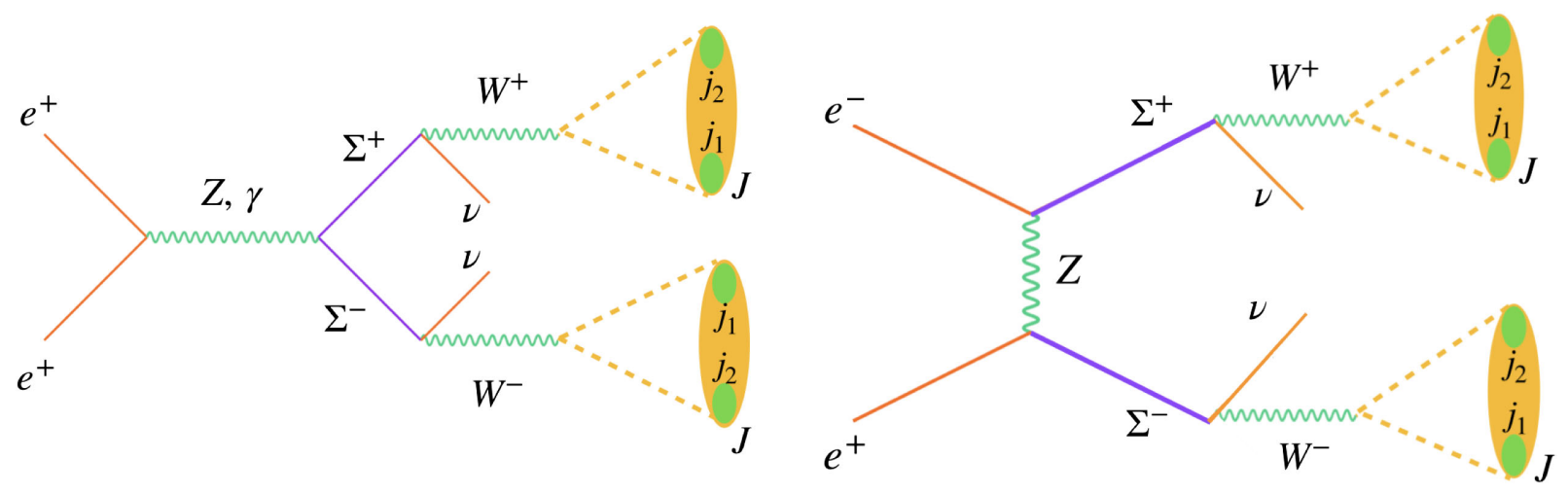

FIG. 17. Fat jet $(J)$ production from the $\Sigma^{ \pm}$at the $e^{+} e^{-}$collider from the $s$-channel process.

leading SM background for two benchmark points $M_{\Sigma}=1 \mathrm{TeV}$ and $2.2 \mathrm{TeV}$, respectively. Note that, as before, these distributions are generated with basic cuts.

Our focus of interest is $800 \mathrm{GeV} \leq M_{\Sigma} \leq 2.9 \mathrm{TeV}$. Based on the normalized distributions in Fig. 16, we have applied following set of advanced selection cuts:

(1) The fat jet transverse momentum is required to be $p_{T}^{J}>150 \mathrm{GeV}$.

(2) The jet mass should be $80 \mathrm{GeV}<m_{J}<100 \mathrm{GeV}$.

(3) The $p_{T}$ of the leading electron is chosen to be $p_{T}^{e_{1}}>350 \mathrm{GeV}$, while $p_{T}$ of the subleading electron, $p_{T}^{e_{2}}$, is assumed to be greater than $200 \mathrm{GeV}$ for $900 \mathrm{GeV} \leq M_{\Sigma} \leq 1.4 \mathrm{TeV}$, greater than $100 \mathrm{GeV}$ for $1.6 \mathrm{TeV} \leq M_{\Sigma} \leq 2.4 \mathrm{TeV}$, and greater than $40 \mathrm{GeV}$ for $2.6 \mathrm{TeV} \leq M_{\Sigma} \leq 3.0 \mathrm{TeV}$.

For the chosen $M_{\Sigma}$ range, the subleading recoils against the $\Sigma^{+}$. Therefore, we found that for the heavier $\Sigma^{+}$the significance improves significantly with lower $p_{T}^{e_{2}}$. The impacts of the event selection cuts are provided in Table VI for $M_{\Sigma}=1 \mathrm{TeV}$ for illustration.

\section{Analysis for the final state $2 J+p_{T}^{\mathrm{miss}}$ at $\sqrt{s}=3 \mathrm{TeV} e^{-} e^{+}$collider}

This final state arises from the pair production of $\Sigma^{+} \Sigma^{-}$ and its subsequent decay $\Sigma^{ \pm} \rightarrow W^{ \pm} \nu, W^{ \pm} \rightarrow J$ at the $e^{-} e^{+}$ collider. The corresponding Feynman diagram is given in Fig. 17. For this final state, the dominant SM backgrounds come from the intermediate processes $W W \nu \nu$ and $W W Z$. In this case, we have considered $800 \mathrm{GeV} \leq M_{\Sigma} \leq 1.4 \mathrm{TeV}$

TABLE VII. Cut flow of the signal and background cross sections for the final state $2 J+p_{T}^{\text {miss }}$ for $M_{\Sigma}=1 \mathrm{TeV}$ at the $\sqrt{s}=3 \mathrm{TeV} e^{-} e^{+}$collider.

\begin{tabular}{lcrrr}
\hline \hline & & \multicolumn{3}{c}{ Background $(\mathrm{fb})$} \\
\cline { 3 - 4 } Cuts & Signal $(\mathrm{fb})$ & $W W \nu \nu$ & $W W Z$ & Total $(\mathrm{fb})$ \\
\hline Basic cuts & 0.341 & 16.659 & 1.406 & 18.065 \\
$p_{T}^{\text {miss }}>200 \mathrm{GeV}$ & 0.303 & 9.251 & 0.941 & 10.191 \\
$p_{T}^{J}>300 \mathrm{GeV}$ & 0.296 & 5.813 & 0.896 & 6.708 \\
$70 \mathrm{GeV} \leq m_{J} \leq 90 \mathrm{GeV}$ & 0.233 & 3.695 & 0.657 & 4.352 \\
\hline \hline
\end{tabular}

at $\sqrt{s}=3 \mathrm{TeV} e^{-} e^{+}$collider. In addition to the basic cuts, we have applied the following set of advanced selection cuts to reduce the SM backgrounds:

(1) Missing energy is $p_{T}^{\text {miss }}>200 \mathrm{GeV}$.

(2) Transverse momentum for the fat jet should be $p_{T}^{J}>300 \mathrm{GeV}$.

(3) The fat jet mass should be $70 \mathrm{GeV} \leq m_{J} \leq 90 \mathrm{GeV}$.

Studying the signal and the backgrounds, we have calculated the significance of the $2 J+p_{T}^{\text {miss }}$ process, and it is shown in the left panel of the Fig. 18 at the $3 \mathrm{TeV} e^{-} e^{+}$ collider. The testing potential of this channel can reach up to more than $5 \sigma$ for 3 and $5 \mathrm{ab}^{-1}$ luminosities; however, the impressive significance well above $3 \sigma$ can reach at $1 \mathrm{ab}^{-1}$ luminosity. ${ }^{1}$

At this point, we mention that we have also studied the $2 J_{b}+e^{-} e^{+}$signal from Fig. 17 in which $\Sigma^{ \pm}$decays into $\ell^{ \pm} h$. Each $h$ boson can further decay into a pair of collimated $b$ jets. The cut flow of the process is shown in Table VII. These collimated $b$ jets form a fat $b$ jet. Using a $70 \% b$-tagging efficiency for the signal and SM backgrounds, we give only the significance of this process just as a reference in right panel of Fig. 18. The significance of this process can prospectively reach above the $5 \sigma$ level at the $1 \mathrm{ab}^{-1}$ luminosity at $3 \mathrm{TeV} e^{-} e^{+}$collider. Higher luminosities at 3 and $5 \mathrm{ab}^{-1}$ can improve the significance of the heavier triplets, leading to a prospect of above $5 \sigma$ significance. In this context, we mention that there is the $J_{b}+e^{-} e^{+}$final state, which can be obtained from the single Higgs production as given in Fig. 13. Because of the single fat $b$ jet, this channel is less efficient compared to the $2 J_{b}+e^{-} e^{+}$signal.

\section{E. Analysis for the final state $e^{ \pm}+J+j_{1}$ at FCC-he}

This final state arises from the production of $j_{1} \Sigma^{0}$ and the subsequent decay of $\Sigma^{0} \rightarrow e^{ \pm} W^{\mp}, W^{\mp} \rightarrow J$ at the $e^{-} p$ collider. The corresponding Feynman diagram is given in Fig. 19. In this case, we consider a $\sqrt{s}=3.46 \mathrm{TeV} e^{-} p$ collider. In this section, we study the visible particles in the

\footnotetext{
${ }^{1}$ We use $S_{\text {sig }}=\frac{S}{\sqrt{S+B}}$ to calculate the significance.
} 

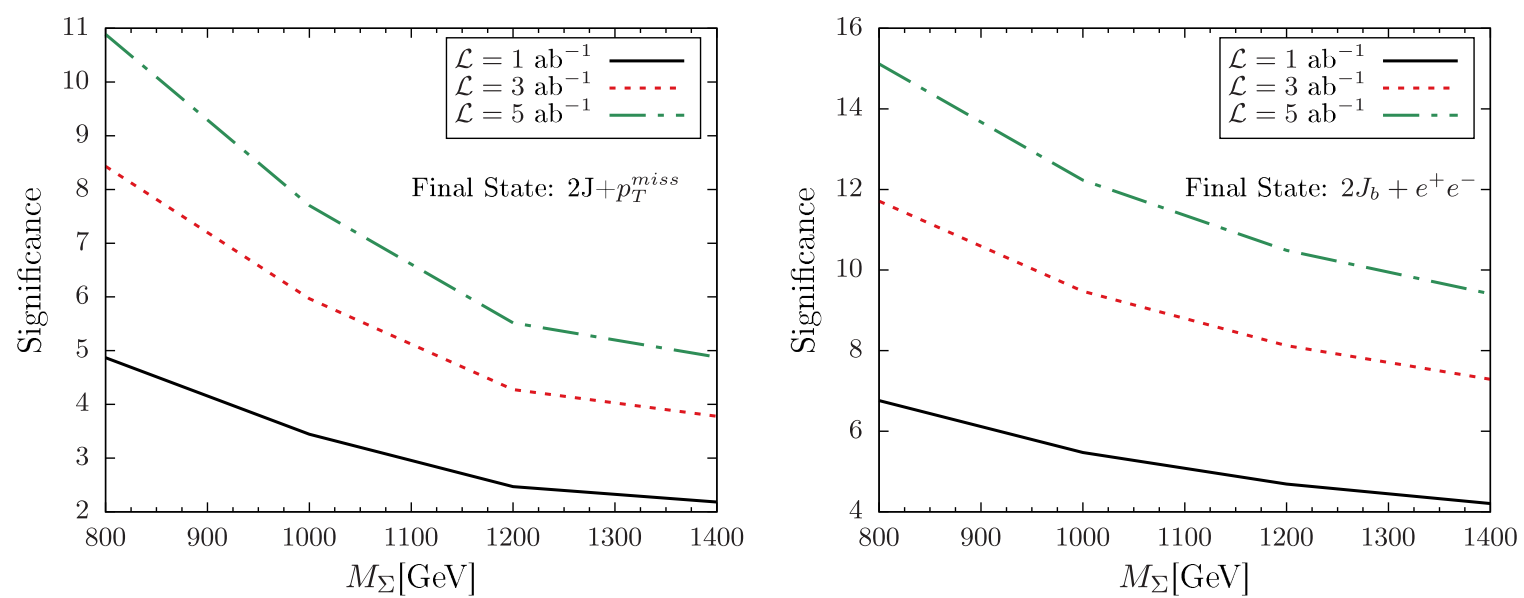

FIG. 18. Signal significance for the final states $2 J+p_{T}^{\text {miss }}$ (left panel) and $2 J_{b}+e^{-} e^{+}$(right panel) from the pair production of $\Sigma^{ \pm}$ with luminosity 1,3 , and $5 \mathrm{ab}^{-1}$, respectively, at the $\sqrt{s}=3 \mathrm{TeV} e^{-} e^{+}$collider.

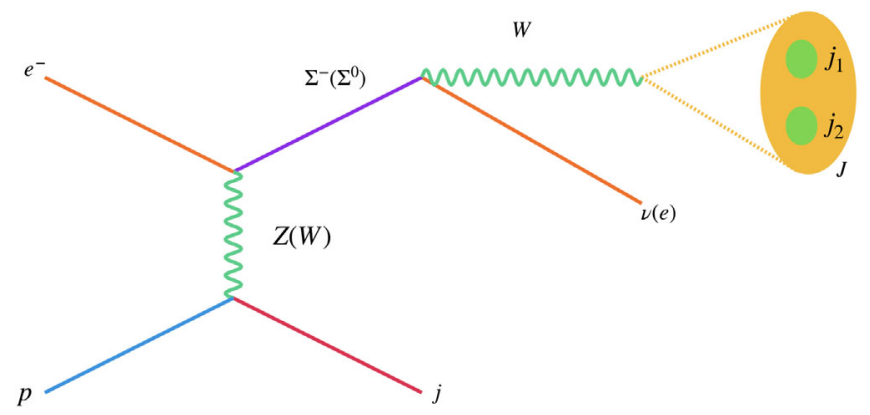

FIG. 19. Fat jet $(J)$ production from the $\Sigma^{-}$and $\Sigma^{0}$ at the $e^{-} p$ collider from the $t$-channel process.

final sate. Here, we have two different processes $e^{+}+J+$ $j_{1}$ and $e^{-}+J+j_{1}$. The first one is a lepton numberviolating (LNV) process, and the second one is lepton number-conserving (LNC) process at the $e^{-} p$ collider. We have combined both these LNV and LNC processes to obtain the final state $e^{ \pm}+J+j_{1}$.

We expect the LNV signal to be almost background free, unless some $e^{+}+$jets events appear from the radiation effects, which one expects to be negligible. For the LNC channel, the dominant SM backgrounds come from the SM processes $e^{-} j j j, e^{-} j j$, and $e^{-} j$ including the initial- and final-state radiations. We have shown the normalized distributions of the leading lepton $p_{T}$, fat jet $p_{T}$, invariant mass of the fat jet, invariant mass of the leading lepton, and fat jet system in Fig. 20. These distributions are for two benchmark points $M_{\Sigma}=1$ and 1.5 TeV. For this final state, we focus in the mass range $800 \mathrm{GeV} \leq M_{\Sigma} \leq 2 \mathrm{TeV}$. In view of these distributions in Fig. 20, we apply the following set of advanced selection cuts:

(1) transverse momentum of fat jet $p_{T}^{J}>200 \mathrm{GeV}$ for $800 \mathrm{GeV} \leq M_{\Sigma} \leq 1.4 \mathrm{TeV}$ and $p_{T}^{J}>400 \mathrm{GeV}$ for $1.5 \mathrm{TeV} M_{\Sigma} \leq 2.0 \mathrm{TeV}$
(2) transverse momentum of lepton $p_{T}^{e^{ \pm}}>200 \mathrm{GeV}$ for $800 \mathrm{GeV} \leq M_{\Sigma} \leq 1.4 \mathrm{TeV}$ and $p_{T}^{e^{ \pm}}>400 \mathrm{GeV}$ for $1.5 \mathrm{TeV} \leq M_{\Sigma} \leq 1.5 \mathrm{TeV}$

(3) fat jet mass $70 \mathrm{GeV} \leq m_{J} \leq 90 \mathrm{GeV}$;

(4) invariant mass window of $\left(e^{ \pm}\right)$and fat jet $(J)$ system, $\left|M_{e J}-M_{\Sigma}\right| \leq 20 \mathrm{GeV}$.

Note that $70 \mathrm{GeV} \leq m_{J} \leq 90 \mathrm{GeV}$ cuts out the SM backgrounds low-energy peaks. Similarly, high $p_{T}$ cuts for the leading lepton and fat jet are extremely useful to reduce the SM backgrounds.

It is difficult to obtain a fat jet for the background process $e j$ because of the $t$-channel exchange of the $Z$ boson and photon. Initial- and final-state radiations can give lowenergy jets, which can produce a soft fat jet. Therefore, the $e j$ background can completely be reduced with high $p_{T}^{e}, p_{T}^{J}$ cuts and fat jet mass $70 \mathrm{GeV} \leq m_{J} \leq 90 \mathrm{GeV}$. These cuts will not be enough to reduce the irreducible backgrounds coming from the process $e j j$ and $e j j j$. However, both of these backgrounds can be reduced using the invariant mass cut of the $\Sigma^{0}$. As $\Sigma^{0}$ decays according to $\Sigma^{0} \rightarrow e W, W \rightarrow J$, the invariant mass of $e J$ system should be close to mass of the $\Sigma^{0}$. Therefore, an invariant mass window cut $\left|M_{e J}-M_{\Sigma}\right| \leq 20 \mathrm{GeV}$ will be extremely useful to reduce these two SM backgrounds further. We have shown the cut flow for two benchmark points $M_{\Sigma}=1$ and $1.5 \mathrm{TeV}$ in Tables VIII and IX.

Further, we also comment that $\Sigma^{-} j$ channel shows a signature of $j_{1}+J+p_{T}^{\text {miss }}$, which is less significant compared to the $j_{1}+e^{ \pm}+J$ channel due to the absence of the visible lepton. The presence of the jets and the missing energy will have more SM backgrounds, which will make the $j_{1}+J+p_{T}^{\text {miss }}$ final state less sensitive.

\section{DISCUSSIONS}

After studying the signals and the SM backgrounds for the triplet fermion production at the $e^{-} e^{+}$collider and $e^{-} p$ 

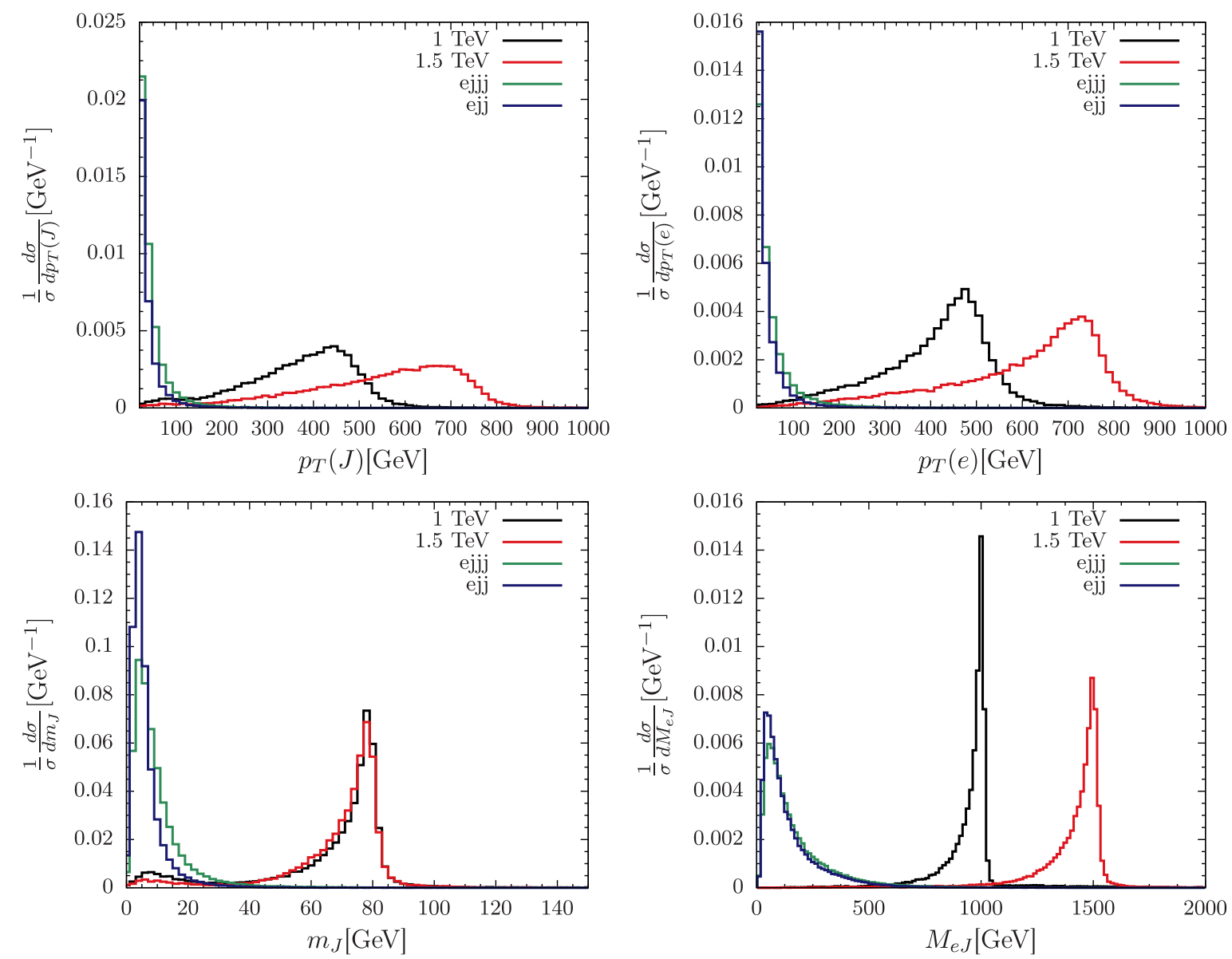

FIG. 20. Normalized fat jet $p_{T}$, leading lepton $p_{T}$, fat jet invariant mass, and lepton distributions for the final state $e^{ \pm}+J+j_{1}$. The black and red lines correspond to signal for $M_{\Sigma}=1$ and $1.5 \mathrm{TeV}$, respectively.

at different energies and luminosities, we calculate the bounds on the mass-mixing plane at $3 \sigma$ and $5 \sigma$ significance. To calculate the bounds on the mixings from the $e^{-} e^{+}$ collider, we use two different center-of-mass energies like 1 and $3 \mathrm{TeV}$. To do the same at the $e^{-} p$ collider, we use 3.46 TeV center-of-mass energy. At these colliders, we have used 1,3 , and $5 \mathrm{ab}^{-1}$ luminosities. We compare our results with the bounds obtained from Ref. [73]. We use the bounds on the mixing $V_{e}=0.019$ and the universal bounds 0.016 as studied from the EWPD. We represent the bounds as EWPD-e and EWPD-U in Figs. 21 and 22 by the horizontal dot-dashed and dotted lines, respectively.

The bounds obtained from the $e^{-} e^{+}$collider studying a variety of final states are shown in Fig. 21. We have studied the $e^{ \pm}+J+p_{T}^{\text {miss }}$ final state from Fig. 7 at the $1 \mathrm{TeV}$ (top, left) and $3 \mathrm{TeV}$ (top, right) $e^{-} e^{+}$colliders. One can probe the mixing up to $8 \times 10^{-5}$ at the $1 \mathrm{TeV}$ collider at $3 \sigma$ significance with the luminosity of $5 \mathrm{ab}^{-1}$. With the same luminosity, the bounds remain below the EWPD-e and EWPD-U up to $900 \mathrm{GeV}$ (EWPD-U) and $945 \mathrm{GeV}$

TABLE VIII. Cut flow of the signal and background cross sections for the final state $e^{ \pm}+J+j_{1}$ for $M_{\Sigma}=1 \mathrm{TeV}$ with $\sqrt{s}=3.46 \mathrm{TeV} e^{-} p$ collider.

\begin{tabular}{lcccc}
\hline \hline & & \multicolumn{2}{c}{ Background $(\mathrm{fb})$} & \\
\cline { 3 - 4 } Cuts & Signal $(\mathrm{fb})$ & $e j j j$ & $e j j$ & Total $(\mathrm{fb})$ \\
\hline Basic cuts & 0.337 & $2.905 \times 10^{5}$ & $5.404 \times 10^{5}$ & $8.309 \times 10^{5}$ \\
$p_{T}^{J}>200 \mathrm{GeV}$ & 0.303 & $2.592 \times 10^{3}$ & $1.799 \times 10^{3}$ & $4.391 \times 10^{3}$ \\
$p_{T}^{e}>200 \mathrm{GeV}$ & 0.294 & $1.891 \times 10^{3}$ & $1.449 \times 10^{3}$ & $3.34 \times 10^{3}$ \\
$70 \mathrm{GeV} \leq m_{J} \leq 90 \mathrm{GeV}$ & 0.202 & 351.808 & 205.075 & 556.883 \\
$\mid M_{e J}-M_{\Sigma} \leq 20 \mathrm{GeV}$ & 0.134 & 9.138 & 4.661 & 13.799 \\
\hline \hline
\end{tabular}


TABLE IX. Cut flow of the signal and background cross sections for the final state $e^{ \pm}+J+j_{1}$ for $M_{\Sigma}=$ $1.5 \mathrm{TeV}$ with $\sqrt{s}=3.46 \mathrm{TeV} e^{-} p$ collider.

\begin{tabular}{|c|c|c|c|c|}
\hline \multirow[b]{2}{*}{ Cuts } & \multirow[b]{2}{*}{ Signal (fb) } & \multicolumn{2}{|c|}{ Background (fb) } & \multirow[b]{2}{*}{ Total (fb) } \\
\hline & & $e j j j$ & $e j j$ & \\
\hline Basic cuts & 0.060 & $2.905 \times 10^{5}$ & $5.404 \times 10^{5}$ & $8.309 \times 10^{5}$ \\
\hline$p_{T}^{J}>400 \mathrm{GeV}$ & 0.048 & 274.136 & 167.788 & 441.924 \\
\hline$p_{T}^{e}>400 \mathrm{GeV}$ & 0.047 & 203.318 & 135.163 & 338.481 \\
\hline $70 \mathrm{GeV} \leq m_{J} \leq 90 \mathrm{GeV}$ & 0.033 & 22.844 & 18.643 & 41.488 \\
\hline$\left|M_{e J}-\bar{M}_{\Sigma}\right| \leq 20 \mathrm{GeV}$ & 0.015 & 0.115 & 0.023 & 0.138 \\
\hline
\end{tabular}

(EWPD-e) at $5 \sigma$ significance. The same has been studied at the $3 \mathrm{TeV} e^{-} e^{+}$collider where the results can impressively be improved even with $1 \mathrm{ab}^{-1}$ luminosity with $5 \sigma$ significance up to $2.5 \mathrm{TeV}$ triplets, which can be further improved at the higher luminosity probing the heavy triplets up to 2.9 TeV. We have studied the $J_{b}+p_{T}^{\text {miss }}$ final state from Fig. 13, and the corresponding bounds at the $3 \mathrm{TeV} e^{-} e^{+}$
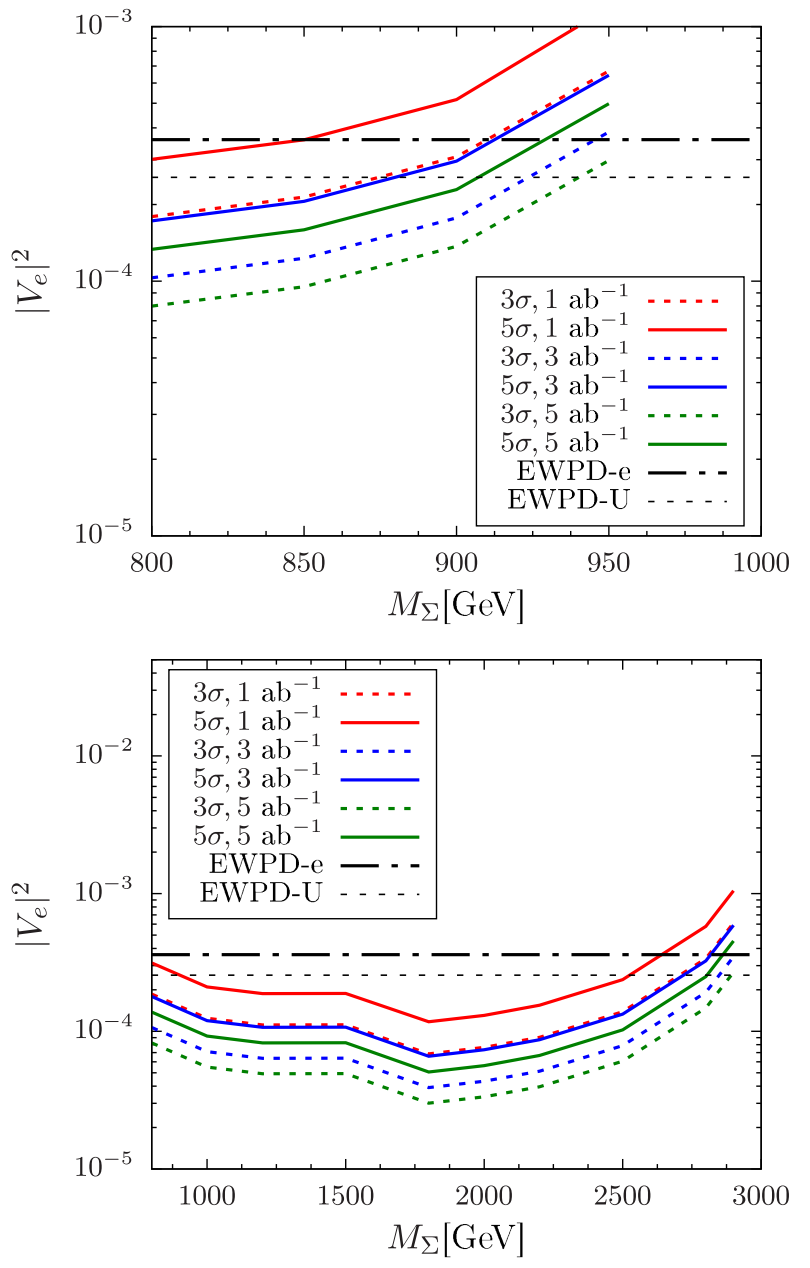

collider are given in Fig. 21 (bottom, left). The lowest mixing $3.5 \times 10^{-5}$ could reach $1.725 \mathrm{TeV}$ heavy triplet mass. At the collider energy threshold, the cross section decreases at the $e^{-} e^{+}$collider, which in turn does not help the further heavier triplets in getting lower bounds; however, analyzing the signal and the corresponding SM backgrounds, we find that slightly heavier triplets than
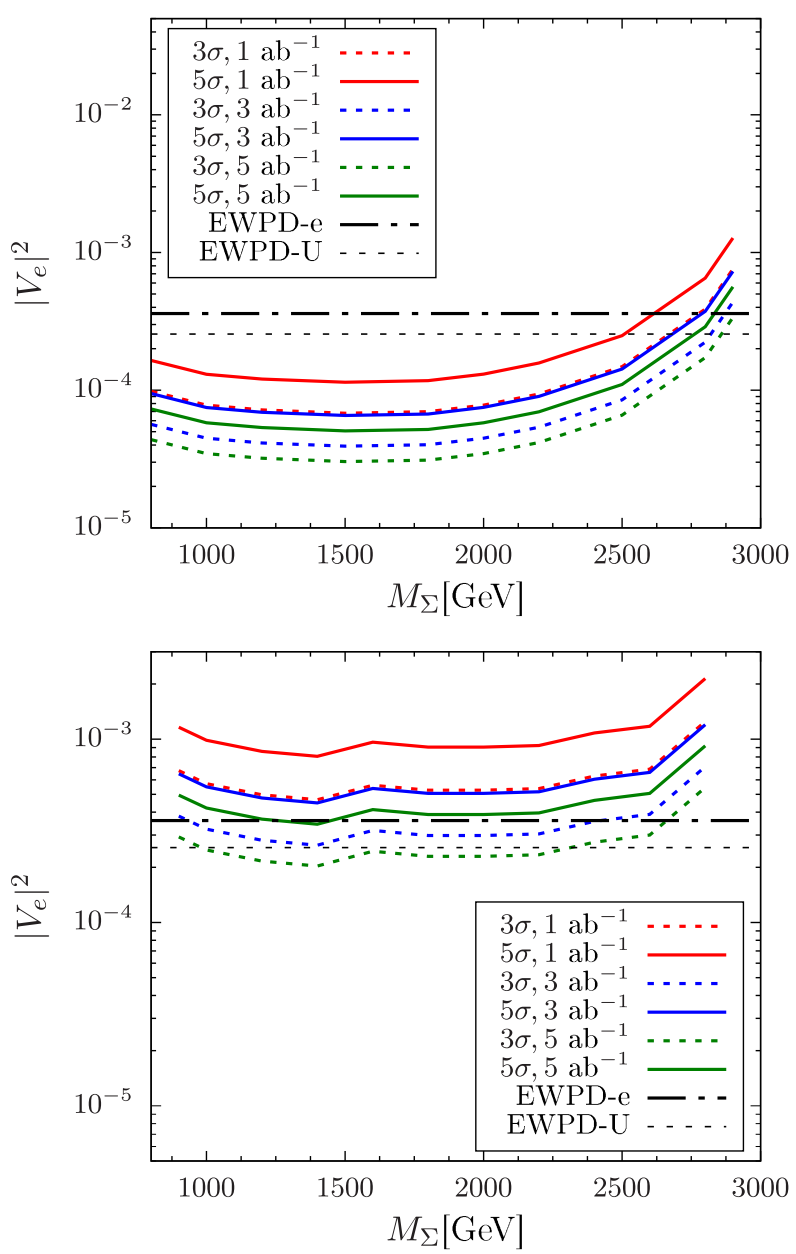

FIG. 21. Sensitivity reach of the mixing angle with the luminosities $\mathcal{L}=1,3$, and $5 \mathrm{ab}^{-1}$ at $3 \sigma$ and $5 \sigma$ significance from the final state $e^{ \pm}+J+p_{T}^{\text {miss }}$ at $\sqrt{s}=1 \mathrm{TeV}$ (top, left) and $\sqrt{s}=3 \mathrm{TeV}$ (top, right), respectively, at the $e^{-} e^{+}$collider. The same for the final states $J_{b}+p_{T}^{\text {miss }}$ (bottom, left) and $e^{+}+e^{-}+J$ (bottom, right), respectively, at the $\sqrt{s}=3 \mathrm{TeV} e^{-} e^{+}$collider with same luminosities like the top panel. The limit from the EWPD for the electron flavor has been represented by the black dot-dashed line from Ref. [73]. 


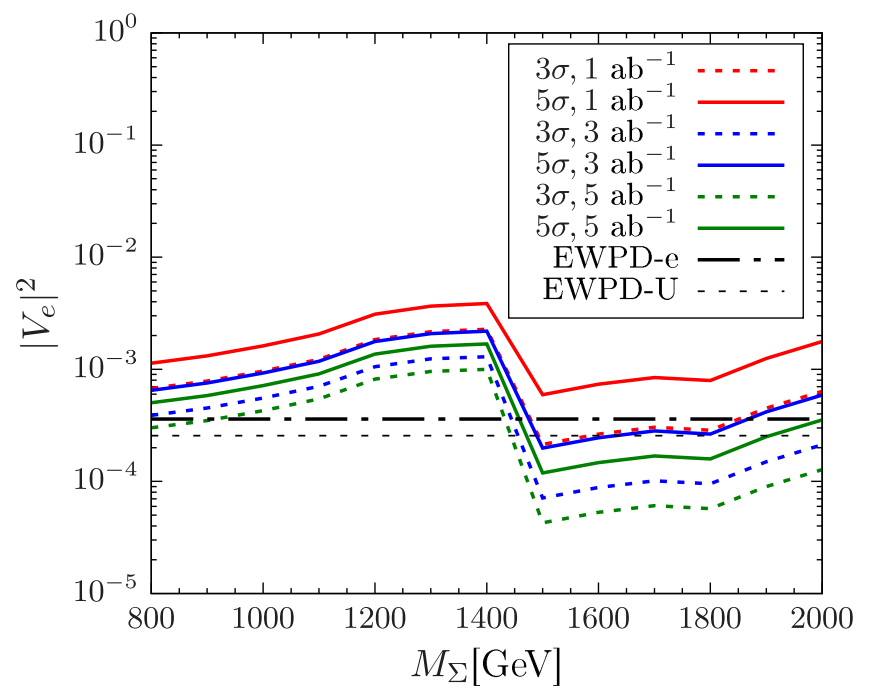

FIG. 22. Sensitivity reach of the mixing angle with the luminosities $\mathcal{L}=1,3$, and $5 \mathrm{ab}^{-1}$ at $3 \sigma$ and $5 \sigma$ significance, from the final state $e^{ \pm}+J+j_{1}$ at $\sqrt{s}=3.46 \mathrm{TeV}$ of the $e^{-} p$ collider.

$2.5 \mathrm{TeV}$ can be probed with $1 \mathrm{ab}^{-1}$ luminosity with $5 \sigma$ significance. Higher luminosity will make the results better. We have studied $e^{+} e^{-}+J$ final state from Fig. 15, and the bound obtained from this final state is shown in Fig. 21 (bottom, right), in which the best results could be obtained at the $3 \mathrm{TeV} e^{-} e^{+}$collider with $5 \mathrm{ab}^{-1}$ luminosity just below the limits obtained from the EWPD at $3 \sigma$ significance.

Bounds obtained from the $e^{-} p$ collider are shown in Fig. 22, studying the $e^{ \pm}+J+j_{1}$ final state from Fig. 19, which has a visible particle in the final state from the leading decay mode compared to the invisible one from the other possibilities. In this case, we find that the heavy triplets are favored to study the fat jets due to their better efficiency for boosting the $W$ boson coming from the leading decay mode of the $\Sigma^{0}$. Comparing our results with the bounds obtained from the EWPD, we find that higher luminosity with 3 and $5 \mathrm{ab}^{-1}$ will be useful to probe heavier triplets. Triplets heavier than $1.4 \mathrm{TeV}$ can be probed up to the mixings $3 \times 10^{-5}$ at $3 \sigma$ significance and $10^{-4}$ at $5 \sigma$ significance at $5 \mathrm{ab}^{-1}$ luminosity. The bounds obtained for $M_{\Sigma}>1.4 \mathrm{TeV}$ are better than those obtained for $M_{\Sigma}<1.4 \mathrm{TeV}$. This is because of the heavier triplets, which produced fat jets better than the comparatively lighter ones.

\section{CONCLUSION}

We have studied the triplet fermion initiated seesaw model, which is commonly known as the type-III seesaw scenario, which is responsible for the light neutrino mass generation through the seesaw mechanism. As a result, a light-heavy mixing appears in the model. We consider the production of the charged and neutral multiplets of the triplet fermion at the $e^{-} e^{+}$and $e^{-} p$ colliders at different center-ofmass energies. Being produced in association with the SM particles and as well as in a pair, the charged and neutral multiplets can decay into a variety of final states.

In this article, we consider mostly the leading decay modes of the triplets and some next-to-leading modes, too. As the mass of the triplet is a free parameter, we consider the heavier mass of the triplet, which can sufficiently boost its decay products. As a result, the decay products including SM gauge bosons and Higgs boson can manifest a fat jet through their leading hadronic decay modes. Finally, we study a variety of final states and the SM backgrounds to probe the light-heavy mixing as a function of the triplet mass for different center-of-mass energies at the abovementioned colliders using different integrated luminosities. Comparing our results with the bounds on the light-heavy mixing obtained from the electroweak precision test results, we find that the heavier triplets can be successfully probed and the bounds on the light-heavy mixing as a function of the triplet mass can be better than the results from the electroweak test.

Apart from the associate production of the triplet, we study the pair production of the heavy triplets at the $e^{-} e^{+}$ collider followed by the decays into the SM bosons. Because of the heavier mass, the triplets can easily boost the daughter particles so that the hadronic decay products of the SM gauge bosons among them can manifest fat jet signatures, which can efficiently segregate the signals from the SM backgrounds leading to the test above $5 \sigma$ significance in the near future.

\section{ACKNOWLEDGMENTS}

The work of A. D. is supported by the JSPS, Grantin-Aid for Scientific Research, Grant No. 18F18321. The work of S.M. is supported by the Spanish Grants No. FPA2017-85216-P (AEI/FEDER, UE) and No. PROMETEO/2018/165 (Generalitat Valenciana) and the Spanish Red Consolider MultiDark FPA2017-90566REDC. The work of T. M. is supported by MOST Grant No. 106-2112-M-002-015-MY3. 
[1] P. Anselmann et al. (GALLEX Collaboration), GALLEX solar neutrino observations: Complete results for GALLEX II, Phys. Lett. B 357, 237 (1995); Erratum, Phys. Lett. B 361, 235 (1995).

[2] Y. Fukuda et al. (Kamiokande Collaboration), Solar Neutrino Data Covering Solar Cycle 22, Phys. Rev. Lett. 77, 1683 (1996).

[3] C. Athanassopoulos et al. (LSND Collaboration), Evidence for $\mathrm{nu}(\mathrm{mu}) \rightarrow \mathrm{nu}(\mathrm{e})$ Neutrino Oscillations from LSND, Phys. Rev. Lett. 81, 1774 (1998).

[4] Y. Fukuda et al. (Super-Kamiokande Collaboration), Measurement of a small atmospheric muon-neutrino/electronneutrino ratio, Phys. Lett. B 433, 9 (1998).

[5] J. N. Abdurashitov et al. (SAGE Collaboration), Results from SAGE, Phys. Lett. B 328, 234 (1994).

[6] Q. Ahmad et al. (SNO Collaboration), Direct Evidence for Neutrino Flavor Transformation from Neutral Current Interactions in the Sudbury Neutrino Observatory, Phys. Rev. Lett. 89, 011301 (2002).

[7] Q. Ahmad et al. (SNO Collaboration), Measurement of Day and Night Neutrino Energy Spectra at SNO and Constraints on Neutrino Mixing Parameters, Phys. Rev. Lett. 89, 011302 (2002).

[8] J. Hosaka et al. (Super-Kamiokande Collaboration), Three flavor neutrino oscillation analysis of atmospheric neutrinos in Super-Kamiokande, Phys. Rev. D 74, 032002 (2006).

[9] K. Eguchi et al. (KamLAND Collaboration), First Results from KamLAND: Evidence for Reactor Anti-Neutrino Disappearance, Phys. Rev. Lett. 90, 021802 (2003).

[10] M. Ahn et al. (K2K Collaboration), Indications of Neutrino Oscillation in a $250 \mathrm{~km}$ Long Baseline Experiment, Phys. Rev. Lett. 90, 041801 (2003).

[11] M. Catanesi (T2K Collaboration), T2K results and perspectives, Nucl. Phys. B, Proc. Suppl. 237-238, 129 (2013).

[12] P. Adamson et al. (MINOS Collaboration), Improved Search for Muon-Neutrino to Electron-Neutrino Oscillations in MINOS, Phys. Rev. Lett. 107, 181802 (2011).

[13] Y. Abe et al. (Double Chooz Collaboration), Indication of Reactor $\bar{\nu}_{e}$ Disappearance in the Double Chooz Experiment, Phys. Rev. Lett. 108, 131801 (2012).

[14] F. An et al. (Daya Bay Collaboration), Observation of Electron-Antineutrino Disappearance at Daya Bay, Phys. Rev. Lett. 108, 171803 (2012).

[15] J. Ahn et al. (RENO Collaboration), Observation of Reactor Electron Antineutrino Disappearance in the RENO Experiment, Phys. Rev. Lett. 108, 191802 (2012).

[16] C. Patrignani et al. (Particle Data Group), Review of particle physics, Chin. Phys. C 40, 100001 (2016).

[17] S. Weinberg, Baryon and Lepton Nonconserving Processes, Phys. Rev. Lett. 43, 1566 (1979).

[18] P. Minkowski, $\mu \rightarrow e \gamma$ at a rate of one out of $10^{9}$ muon decays? Phys. Lett. B 67, 421 (1977).

[19] R. N. Mohapatra and G. Senjanovic, Neutrino Mass and Spontaneous Parity Nonconservation, Phys. Rev. Lett. 44, 912 (1980).

[20] J. Schechter and J. Valle, Neutrino masses in $\mathrm{SU}(2) \times \mathrm{U}(1)$ theories, Phys. Rev. D 22, 2227 (1980).

[21] T. Yanagida, Horizontal gauge symmetry and masses of neutrinos, Conf. Proc. C7902131, 95 (1979).
[22] M. Gell-Mann, P. Ramond, and R. Slansky, Complex spinors and unified theories, Conf. Proc. C790927, 315 (1979).

[23] S. Glashow, The future of elementary particle physics, NATO Sci. Ser. B 61, 687 (1980).

[24] R. Foot, H. Lew, X. He, and G. C. Joshi, Seesaw neutrino masses induced by a triplet of leptons, Z. Phys. C 44, 441 (1989).

[25] F. del Aguila and J. Aguilar-Saavedra, Electroweak scale seesaw and heavy Dirac neutrino signals at LHC, Phys. Lett. B 672, 158 (2009).

[26] F. del Aguila and J. Aguilar-Saavedra, Distinguishing seesaw models at LHC with multi-lepton signals, Nucl. Phys. B813, 22 (2009).

[27] R. Franceschini, T. Hambye, and A. Strumia, Type-III seesaw at LHC, Phys. Rev. D 78, 033002 (2008).

[28] C. Biggio and F. Bonnet, Implementation of the type III seesaw model in FeynRules/MADGRAPH and prospects for discovery with early LHC data, Eur. Phys. J. C 72, 1899 (2012).

[29] P. Bandyopadhyay, S. Choi, E. J. Chun, and K. Min, Probing Higgs bosons via the type III seesaw mechanism at the LHC, Phys. Rev. D 85, 073013 (2012).

[30] R. Ruiz, QCD corrections to pair production of type III seesaw leptons at hadron colliders, J. High Energy Phys. 12 (2015) 165.

[31] D. Goswami and P. Poulose, Direct searches of type III seesaw triplet fermions at high energy $e^{+} e^{-}$collider, Eur. Phys. J. C 78, 42 (2018).

[32] S. Jana, N. Okada, and D. Raut, Displaced vertex and disappearing track signatures in type-III seesaw, arXiv: 1911.09037.

[33] E. Ma, Pathways to Naturally Small Neutrino Masses, Phys. Rev. Lett. 81, 1171 (1998).

[34] S. Dimopoulos and H. Georgi, Softly broken supersymmetry and SU(5), Nucl. Phys. B193, 150 (1981).

[35] N. Sakai, Naturalness in supersymmetric guts, Z. Phys. C 11, 153 (1981).

[36] B. Bajc and G. Senjanovic, Seesaw at LHC, J. High Energy Phys. 08 (2007) 014.

[37] P. Fileviez Perez, Renormalizable adjoint SU(5), Phys. Lett. B 654, 189 (2007).

[38] P. Fileviez Perez, Supersymmetric adjoint SU(5), Phys. Rev. D 76, 071701 (2007).

[39] R. Adhikari, J. Erler, and E. Ma, Seesaw neutrino mass and new U(1) gauge symmetry, Phys. Lett. B 672, 136 (2009).

[40] A. Arhrib, B. Bajc, D. K. Ghosh, T. Han, G.-Y. Huang, I. Puljak, and G. Senjanovic, Collider signatures for heavy lepton triplet in type I + III seesaw, Phys. Rev. D 82, 053004 (2010).

[41] B. Bajc, M. Nemevsek, and G. Senjanovic, Probing leptonic CP phases in LFV processes, Phys. Lett. B 684, 231 (2010).

[42] D. Ibanez, S. Morisi, and J. Valle, Inverse tri-bimaximal type-III seesaw and lepton flavor violation, Phys. Rev. D 80, 053015 (2009).

[43] E. Ma, Inverse seesaw neutrino mass from lepton triplets in the U(1)(Sigma) model, Mod. Phys. Lett. A 24, 2491 (2009).

[44] S. M. Barr, B. Bednarz, and C. Benesh, Anomaly constraints and new U(1) gauge bosons, Phys. Rev. D 34, 235 (1986). 
[45] E. Ma, New U(1) gauge symmetry of quarks and leptons, Mod. Phys. Lett. A 17, 535 (2002).

[46] S. M. Barr and I. Dorsner, The origin of a peculiar extra U(1), Phys. Rev. D 72, 015011 (2005).

[47] J. Chakrabortty, A. Dighe, S. Goswami, and S. Ray, Renormalization group evolution of neutrino masses and mixing in the type-III seesaw mechanism, Nucl. Phys. B820, 116 (2009).

[48] I. Gogoladze, N. Okada, and Q. Shafi, Higgs boson mass bounds in the Standard Model with type III and type I seesaw, Phys. Lett. B 668, 121 (2008).

[49] S. Goswami, K. Vishnudath, and N. Khan, Constraining the minimal type-III seesaw model with naturalness, lepton flavor violation, and electroweak vacuum stability, Phys. Rev. D 99, 075012 (2019).

[50] E. Ma and D. Roy, Heavy triplet leptons and new gauge boson, Nucl. Phys. B644, 290 (2002).

[51] CMS Collaboration, Search for type III seesaw from pp collisions at $7 \mathrm{TeV}$, https://inspirehep.net/literature/1260781.

[52] S. Chatrchyan et al. (CMS Collaboration), Search for heavy lepton partners of neutrinos in proton-proton collisions in the context of the type III seesaw mechanism, Phys. Lett. B 718, 348 (2012).

[53] CMS Collaboration, Search for heavy lepton partners of neutrinos in pp collisions at $8 \mathrm{TeV}$, in the context of type III seesaw mechanism, https://inspirehep.net/literature/1351986.

[54] CMS Collaboration, Search for type-III seesaw heavy fermions with multilepton final states using $2.3 / \mathrm{fb}$ of $13 \mathrm{TeV}$ proton-proton collision data, https://inspirehep .net/literature/1434349.

[55] A. M. Sirunyan et al. (CMS Collaboration), Search for Evidence of the Type-III Seesaw Mechanism in Multilepton Final States in Proton-Proton Collisions at $\sqrt{s}=13 \mathrm{TeV}$, Phys. Rev. Lett. 119, 221802 (2017).

[56] CMS Collaboration, Search for evidence of type-III seesaw mechanism in multilepton final states in pp collisions at $\sqrt{s}=13 \mathrm{TeV}$, https://inspirehep.net/literature/1518839.

[57] CMS Collaboration, Search for new physics in multilepton final states in $\mathrm{pp}$ collisions at $\sqrt{s}=13 \mathrm{TeV}$, https:// inspirehep.net/literature/1726693.

[58] G. Aad et al. (ATLAS Collaboration), Search for type-III seesaw heavy leptons in $p p$ collisions at $\sqrt{s}=8 \mathrm{TeV}$ with the ATLAS detector, Phys. Rev. D 92, 032001 (2015).

[59] ATLAS Collaboration, Search for type-III seesaw heavy leptons in proton-proton collisions at $\sqrt{s}=13 \mathrm{TeV}$ with the ATLAS detector, https://cds.cern.ch/record/2621484.

[60] A. Abada, C. Biggio, F. Bonnet, M. Gavela, and T. Hambye, $\mu \rightarrow e \gamma$ and $\tau \rightarrow \ell \gamma$ decays in the fermion triplet seesaw model, Phys. Rev. D 78, 033007 (2008).

[61] A. Abada, C. Biggio, F. Bonnet, M. Gavela, and T. Hambye, Low energy effects of neutrino masses, J. High Energy Phys. 12 (2007) 061.

[62] C. Biggio, E. Fernandez-Martinez, M. Filaci, J. HernandezGarcia, and J. Lopez-Pavon, Global bounds on the type-III seesaw, J. High Energy Phys. 05 (2020) 022.

[63] S. Antusch, C. Biggio, E. Fernandez-Martinez, M. Gavela, and J. Lopez-Pavon, Determining the PMNS Matrix Elements without Assuming Unitarity, AIP Conf. Proc. 903, 279 (2007).
[64] D. Forero, S. Morisi, M. Tortola, and J. Valle, Lepton flavor violation and non-unitary lepton mixing in low-scale type-I seesaw, J. High Energy Phys. 09 (2011) 142.

[65] L. Basso, O. Fischer, and J. J. van der Bij, Precision tests of unitarity in leptonic mixing, Europhys. Lett. 105, 11001 (2014).

[66] S. Antusch and O. Fischer, Non-unitarity of the leptonic mixing matrix: Present bounds and future sensitivities, J. High Energy Phys. 10 (2014) 094.

[67] E. Fernandez-Martinez, J. Hernandez-Garcia, J. LopezPavon, and M. Lucente, Loop level constraints on seesaw neutrino mixing, J. High Energy Phys. 10 (2015) 130.

[68] S. Antusch and O. Fischer, Probing the nonunitarity of the leptonic mixing matrix at the CEPC, Int. J. Mod. Phys. A 31, 1644006 (2016).

[69] C. S. Fong, H. Minakata, and H. Nunokawa, Non-unitary evolution of neutrinos in matter and the leptonic unitarity test, J. High Energy Phys. 02 (2019) 015.

[70] J. Hernandez-Garcia and J. Lopez-Pavon, Non-Unitarity vs sterile neutrinos at DUNE, in Proceedings, Prospects in Neutrino Physics (NuPhys2016): London, UK, 2016 (2017), arXiv:1705.01840.

[71] F. Escrihuela, L. Flores, and O. Miranda, Neutrino counting experiments and non-unitarity from LEP and future experiments, Phys. Lett. B 802, 135241 (2020).

[72] M. Raidal et al., Flavour physics of leptons and dipole moments, Eur. Phys. J. C 57, 13 (2008).

[73] F. del Aguila, J. de Blas, and M. Perez-Victoria, Effects of new leptons in Electroweak Precision Data, Phys. Rev. D 78, 013010 (2008).

[74] J. Gluza and M. Zralek, On possibility of detecting the e- e- $\longrightarrow$ W- W- process in the standard model, Phys. Lett. B 362, 148 (1995).

[75] J. Gluza and M. Zralek, Is there a chance to find heavy neutrinos in future linear colliders?, Phys. Lett. B 372, 259 (1996).

[76] J. Gluza, J. Maalampi, M. Raidal, and M. Zralek, Heavy neutrino mixing and single production at linear collider, Phys. Lett. B 407, 45 (1997).

[77] A. Das and N. Okada, Inverse seesaw neutrino signatures at the LHC and ILC, Phys. Rev. D 88, 113001 (2013).

[78] S. Banerjee, P. S. B. Dev, A. Ibarra, T. Mandal, and M. Mitra, Prospects of heavy neutrino searches at future lepton colliders, Phys. Rev. D 92, 075002 (2015).

[79] S. Antusch, E. Cazzato, and O. Fischer, Higgs production from sterile neutrinos at future lepton colliders, J. High Energy Phys. 04 (2016) 189.

[80] S. Antusch, E. Cazzato, and O. Fischer, Displaced vertex searches for sterile neutrinos at future lepton colliders, J. High Energy Phys. 12 (2016) 007.

[81] S. Antusch, E. Cazzato, and O. Fischer, Sterile neutrino searches at future $e^{-} e^{+}, p p$, and $e^{-} p$ colliders, Int. J. Mod. Phys. A 32, 1750078 (2017).

[82] A. Das and N. Okada, Bounds on heavy Majorana neutrinos in type-I seesaw and implications for collider searches, Phys. Lett. B 774, 32 (2017).

[83] S. Chakraborty, M. Mitra, and S. Shil, Fat jet signature of a heavy neutrino at lepton collider, Phys. Rev. D 100, 015012 (2019). 
[84] A. Das, S. Jana, S. Mandal, and S. Nandi, Probing right handed neutrinos at the $\mathrm{LHeC}$ and lepton colliders using fat jet signatures, Phys. Rev. D 99, 055030 (2019).

[85] S. Mondal and S. K. Rai, Polarized window for left-right symmetry and a right-handed neutrino at the large hadronelectron collider, Phys. Rev. D 93, 011702 (2016).

[86] S. Mondal and S. K. Rai, Probing the heavy neutrinos of inverse seesaw model at the LHeC, Phys. Rev. D 94, 033008 (2016).

[87] S. Mandal, M. Mitra, and N. Sinha, Probing leptoquarks and heavy neutrinos at the LHeC, Phys. Rev. D 98, 095004 (2018).

[88] M. Cirelli, N. Fornengo, and A. Strumia, Minimal dark matter, Nucl. Phys. B753, 178 (2006).

[89] M. Tanabashi et al. (Particle Data Group), Review of particle physics, Phys. Rev. D 98, 030001 (2018).

[90] A. Alloul, N. D. Christensen, C. Degrande, C. Duhr, and B. Fuks, FeynRules2.0-A complete toolbox for tree-level phenomenology, Comput. Phys. Commun. 185, 2250 (2014).

[91] J. Alwall, R. Frederix, S. Frixione, V. Hirschi, F. Maltoni, O. Mattelaer, H.-S. Shao, T. Stelzer, P. Torrielli, and M. Zaro, The automated computation of tree-level and next-toleading order differential cross sections, and their matching to parton shower simulations, J. High Energy Phys. 07 (2014) 079.

[92] T. Sjostrand, S. Mrenna, and P.Z. Skands, PYTHIA6.4 physics and manual, J. High Energy Phys. 05 (2006) 026.

[93] T. Sjöstrand, S. Ask, J. R. Christiansen, R. Corke, N. Desai, P. Ilten, S. Mrenna, S. Prestel, C. O. Rasmussen, and P. Z. Skands, An introduction to PYTHIA8.2, Comput. Phys. Commun. 191, 159 (2015).

[94] J. de Favereau et al. (DELPHES3 Collaboration), DELPHES3, A modular framework for fast simulation of a generic collider experiment, J. High Energy Phys. 02 (2014) 057.

[95] Conference talk by O. Fischer, Exotic Higgs search at Higgs \& top at LHeC, https://indico.cern.ch/event/774889/.

[96] Y. L. Dokshitzer, G. Leder, S. Moretti, and B. Webber, Better jet clustering algorithms, J. High Energy Phys. 08 (1997) 001.

[97] M. Wobisch and T. Wengler, Hadronization corrections to jet cross-sections in deep inelastic scattering, arXiv:hep-ph/ 9907280.

[98] M. Cacciari and G. P. Salam, Dispelling the $N^{3}$ myth for the $k_{t}$ jet-finder, Phys. Lett. B 641, 57 (2006).

[99] M. Cacciari, G. P. Salam, and G. Soyez, FastJet user manual, Eur. Phys. J. C 72, 1896 (2012). 